\title{
Stability of spikes in the shadow Gierer-Meinhardt system with Robin boundary conditions
}

\author{
Philip K. Maini \\ Centre for Mathematical Biology, Mathematical Institute, University of Oxford, 24-29 St Giles', \\ Oxford OX1 3LB, United Kingdom and Department of Biochemistry, Oxford Centre for Integrative Systems \\ Biology, University of Oxford, South Parks Road, Oxford OX1 3QU, United Kingdom \\ Juncheng Wei \\ Department of Mathematics, The Chinese University of Hong Kong, Shatin, Hong Kong \\ Matthias Winter \\ Department of Mathematical Sciences, Brunel University, Uxbridge UB8 3PH, United Kingdom
}

(Received 19 January 2007; accepted 10 July 2007; published online 28 September 2007)

We consider the shadow system of the Gierer-Meinhardt system in a smooth bounded domain $\Omega \subset R^{N}, A_{t}=\epsilon^{2} \Delta A-A+\frac{A^{p}}{\xi^{q}}, x \in \Omega, t>0, \tau|\Omega| \xi_{t}=-|\Omega| \xi+\frac{1}{\xi s} \int_{\Omega} A^{r} d x, t>0$ with the Robin boundary condition $\epsilon \partial A / \partial \nu+a_{A} A=0, x \in \partial \Omega$, where $a_{A}>0$, the reaction rates $(p, q, r, s)$ satisfy $1<p$ $<\left(\frac{N+2}{N-2}\right)_{+}, q>0, r>0, s \geq 0,1<\frac{q r}{(s+1)(p-1)}<+\infty$, the diffusion constant is chosen such that $\epsilon \ll 1$, and the time relaxation constant is such that $\tau \geq 0$. We rigorously prove the following results on the stability of one-spike solutions: (i) If $r=2$ and $1<p<1+4 / N$ or if $r=p+1$ and $1<p<\infty$, then for $a_{A}>1$ and $\tau$ sufficiently small the interior spike is stable. (ii) For $N=1$ if $r=2$ and $1<p \leq 3$ or if $r=p+1$ and $1<p<\infty$, then for $0<a_{A}<1$ the near-boundary spike is stable. (iii) For $N=1$ if 3 $<p<5$ and $r=2$, then there exist $a_{0} \in(0,1)$ and $\mu_{0}>1$ such that for $a \in\left(a_{0}, 1\right)$ and $\mu$ $=2 q /(s+1)(p-1) \in\left(1, \mu_{0}\right)$ the near-boundary spike solution is unstable. This instability is not present for the Neumann boundary condition but only arises for the Robin boundary condition. Furthermore, we show that the corresponding eigenvalue is of order $O(1)$ as $\epsilon \rightarrow 0$. C 2007 American Institute of Physics. [DOI: 10.1063/1.2768156]

Boundary conditions play an important role for pattern formation in Turing systems. In most investigations Neumann (no-flux) boundary conditions have been considered to model an impermeable membrane. However, for some biological applications such as the modeling of skeletal limb development, this does not represent the true biological situation and Robin (mixed) boundary conditions are more realistic. ${ }^{1}$ In this paper, we show that for Robin boundary conditions, some patterns that are stable for Neumann boundary conditions become unstable due to the presence of a new instability. In particular, we are interested in spikes and our main results says that, for the shadow Gierer-Meinhardt system, a spike near the boundary may be destabilized by the change of boundary conditions. This implies that some patterns become more robust at the expense of others that turn unstable. Results of this type are important to understand the role of the boundary conditions in pattern selection.

\section{INTRODUCTION}

Since the work of Turing ${ }^{2}$ in 1952, many models have been established and investigated to explore the instability of homogeneous steady states, which is now called Turing instability. One of the most famous models in biological pattern formation is the Gierer-Meinhardt system. ${ }^{3-5}$ It can be stated as follows:

$$
\begin{aligned}
& A_{t}=\epsilon^{2} \Delta A-A+\frac{A^{p}}{H^{q}}, \quad x \in \Omega, t>0, \\
& \tau H_{t}=D \Delta H-H+\frac{A^{r}}{H^{s}}, \quad x \in \Omega, t>0,
\end{aligned}
$$

where $\Omega \subset R^{N}$ is a bounded, smooth domain. Further, we assume that the reaction exponents $(p, q, r, s)$ satisfy

$$
\begin{aligned}
& 1<p<\left(\frac{N+2}{N-2}\right)_{+}, \quad q>0, r>0, s \geq 0, \\
& 1<\frac{q r}{(s+1)(p-1)}<+\infty
\end{aligned}
$$

where

$$
\left(\frac{N+2}{N-2}\right)_{+}= \begin{cases}+\infty & \text { for } N=1,2 \\ \frac{N+2}{N-2} & \text { for } N=3,4, \ldots .\end{cases}
$$

We assume that the first diffusion constant satisfies $\epsilon \ll 1$ and we will consider the case of $D=\infty$, the so-called shadow system of the Gierer-Meinhardt system. The time relaxation constant is chosen such that $\tau \geq 0$ is independent of $\epsilon$. 
This is a typical activator-inhibitor system, where $A$ denotes an activator and $H$ denotes an inhibitor. This model has been extensively studied in recent years, usually with Neumann boundary conditions. ${ }^{6-12}$

In this work, we consider Robin boundary conditions (also called mixed boundary conditions), which can be stated as follows:

$$
\epsilon \frac{\partial A}{\partial \nu}+a_{A} A=0, \quad \sqrt{D} \frac{\partial H}{\partial \nu}+a_{H} H=0, \quad x \in \partial \Omega,
$$

where $a_{A}>0, a_{H} \geq 0$.

Reaction-diffusion systems have been studied mostly with Neumann boundary conditions. From a biological viewpoint, such boundary conditions correspond to an impermeable membrane/barrier. While in many cases this is a realistic assumption, there are several applications, for example in skeletal limb development, where the boundary is a source of some chemical morphogens and a sink for others. It is therefore essential that the study of reaction-diffusion systems is extended to incorporate more general types of boundary conditions. For example, in Ref. 1 a comparative numerical study of a reaction-diffusion system coming from glycolysis with a range of different boundary conditions revealed that certain types of boundary conditions selected particular patterning modes at the expense of others. It was also shown that the robustness of certain patterns could be greatly enhanced and the authors showed a possible application to skeletal patterns in the limb. This study in Ref. 1 answered the standard criticism of Turing patterns being too sensitive to fluctuations for the model to be viable for robust embryological patterning.

In this paper, we take a first step in this direction. Instead of studying multicomponent reaction-diffusion systems, we initiate a rigorous study of stationary spikes for the shadow system of the Gierer-Meinhardt system, given in Eqs. (1.1) and (1.2).

We now (formally) derive the shadow system of Eqs. (1.1) and (1.2). To this end, we let $D \rightarrow \infty$ and suppose that $A$ and $H$ remain bounded. Then

$$
\Delta H \rightarrow 0 \quad \text { in } \Omega
$$

and

$$
\frac{\partial H}{\partial \nu} \rightarrow 0 \quad \text { on } \partial \Omega
$$

This implies that $H(x) \rightarrow \xi$, a constant in $\Omega$ that might depend on time $t$. To derive the equation for $\xi$, we integrate both sides of the second equation in (1.1) over $\Omega$. For the left-hand side, we obtain

$$
\tau \int_{\Omega} H_{t}(x) d x=\tau\left(\int_{\Omega} H(x) d x\right)_{t} \rightarrow \tau|\Omega| \xi_{t} .
$$

To compute the right-hand side, we begin with

$$
\begin{aligned}
D \int_{\Omega} \Delta H(x) d x & =D \int_{\partial \Omega} \frac{\partial H}{\partial \nu}(x) d S \\
& =\sqrt{D} \int_{\partial \Omega}\left(-a_{H}\right) H(x) d S \\
& \rightarrow-\sqrt{D} a_{H}|\partial \Omega| \xi,
\end{aligned}
$$

where we have used (1.2). Further, we compute

$$
\int_{\Omega} H(x) d x \rightarrow|\Omega| \xi
$$

and

$$
\int_{\Omega} \frac{A^{r}(x)}{H^{s}(x)} d x \rightarrow \int_{\Omega} \frac{A^{r}(x)}{\xi^{s}} d x=\frac{\int_{\Omega} A^{r}(x) d x}{\xi^{s}} .
$$

From these computations, we finally get the following socalled shadow system of Eq. (1.1):

$$
\begin{aligned}
& A_{t}=\epsilon^{2} \Delta A-A+\frac{A^{p}}{\xi^{q}}, \quad x \in \Omega, \\
& t>0, \tau|\Omega| \xi_{t}=-\left(|\Omega|+\sqrt{D} a_{H}|\partial \Omega|\right) \xi+\frac{1}{\xi^{s}} \int_{\Omega} A^{r} d x, \\
& \quad x \in \Omega, t>0, \\
& \epsilon \frac{\partial A}{\partial \nu}+a_{A} A=0, \quad x \in \partial \Omega, t>0 .
\end{aligned}
$$

For simplicity, from now on we consider the case $a_{H}=0$. This means we study the shadow system with the Neumann boundary condition for the inhibitor, which can be stated as follows:

$$
\begin{aligned}
& A_{t}=\epsilon^{2} \Delta A-A+\frac{A^{p}}{\xi^{q}}, \quad x \in \Omega, t>0, \\
& \tau|\Omega| \xi_{t}=-|\Omega| \xi+\frac{1}{\xi^{s}} \int_{\Omega} A^{r} d x, \quad t>0, \\
& \epsilon \frac{\partial A}{\partial \nu}+a_{A} A=0, \quad x \in \partial \Omega, t>0 .
\end{aligned}
$$

Let us now consider stationary solutions to the shadow system (1.3). Set $A(x)=\xi^{q /(p-1)} u(x), a_{A}=a$. Then $u$ satisfies

$$
\begin{aligned}
& \epsilon^{2} \Delta u-u+u^{p}=0 \quad \text { for } x \in \Omega, \\
& u>0 \quad \text { for } x \in \Omega, \\
& \epsilon \frac{\partial u}{\partial \nu}+a u=0 \quad \text { for } x \in \partial \Omega .
\end{aligned}
$$

For $\xi$, we have the equation

$$
0=-|\Omega| \xi+\frac{\xi^{q r /(p-1)}}{\xi^{s}} \int_{\Omega} u^{r} d x,
$$

which gives 

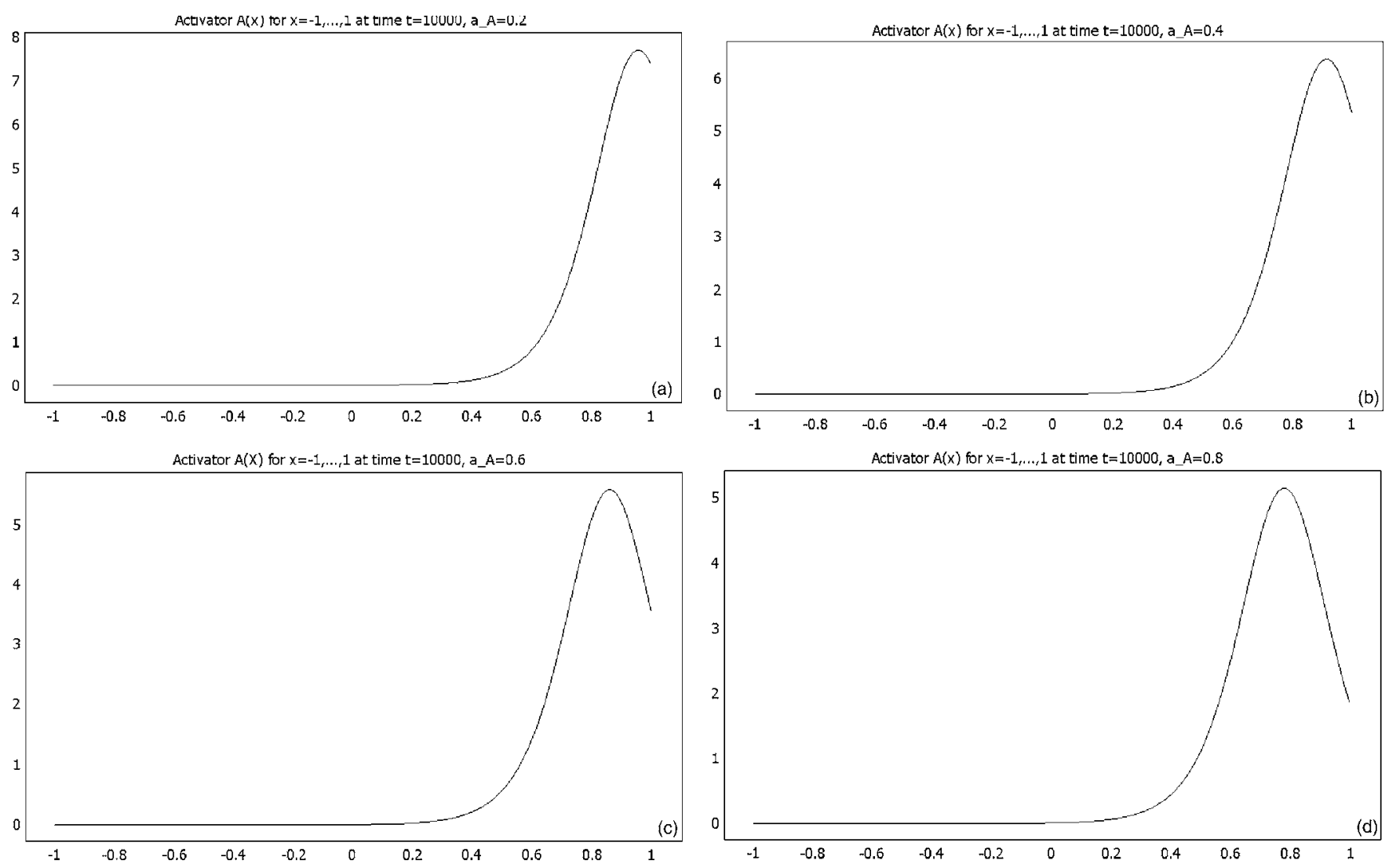

FIG. 1. Near-boundary spike for variable constant $a_{A}$ in the Robin boundary. We have chosen $a_{A}=0.2,0.4,0.6,0.8$. It is numerically stable (final state is shown for $t=10000)$.

$$
\xi^{1+s-q r /(p-1)}=\frac{1}{|\Omega|} \int_{\Omega} u^{r} d x
$$

and so

$$
\xi=\left(\frac{1}{|\Omega|} \int_{\Omega} u^{r} d x\right)^{-(p-1) /[q r-(p-1)(s+1)]}
$$

Problem (1.4) has been studied by Berestycki and Wei in Ref. 20 and the following result has been proved:

Theorem A. Let $1<p<\left(\frac{N+2}{N-2}\right)_{+}$. Then there exists a number $a(N, p)$, where $a(1, p)=1$ and $a(N, p)>1$ for $N \geq 2$, such that problem (1.4) has a solution $u_{\epsilon, a}$ satisfying i.e.,

(1) $u_{\epsilon, a}$ has the least energy among all solutions to (1.4),

$$
E_{\epsilon}\left[u_{\epsilon, a}\right] \leq E_{\epsilon}[u]
$$

for all solutions $u$ to (1.4), where $E_{\epsilon}$ is the energy functional defined by

$$
\begin{aligned}
E_{\epsilon}[u]= & \frac{\epsilon^{2}}{2} \int_{\Omega}|\nabla u|^{2} d x+\frac{1}{2} \int_{\Omega} u^{2} d x-\frac{1}{p+1} \int_{\Omega} u^{p+1} d x \\
& +\frac{\epsilon a}{2} \int_{\partial \Omega} u^{2} d s,
\end{aligned}
$$

where $u_{+}=\max \{u, 0\}$.

(2) If $0<a<a(N, p)$, then $u_{\epsilon, a}$ has a local maximum point $x_{\epsilon} \in \Omega$ with

$$
\frac{d\left(x_{\epsilon}, \partial \Omega\right)}{\epsilon} \rightarrow d_{0}>0 .
$$

(3) If $a>a(N, p)$, then $u_{\epsilon, a}$ has a unique local maximum point $x_{\epsilon} \in \Omega$ with

$$
d\left(x_{\epsilon}, \partial \Omega\right) \rightarrow \max _{x \in \Omega} d(x, \partial \Omega) .
$$

Remark 1.1. (1) The solution in part (2) of Theorem A is called a near-boundary spike (see Fig. 1 in Sec. IV).

(2) The solution in part (3) of Theorem A is called an interior spike (see Fig. 2 in Sec. IV).

(3) We remark that in case (2) of Theorem A, i.e., for 0 $<a<a(N, p)$, there also exists an interior spike that is a solution of (1.4) but does not minimize (1.5) among the solutions of (1.4).

Now we consider the stability of the steady state $\left(A_{\epsilon, a}, \xi_{\epsilon, a}\right)$ to the shadow system (1.3), where

$$
\begin{aligned}
& A_{\epsilon, a}=\xi_{\epsilon, a}^{q /(p-1)} u_{\epsilon, a}, \\
& \xi_{\epsilon, a}=\left(\frac{1}{|\Omega|} \int_{\Omega} u_{\epsilon, a}^{r} d x\right)^{-(p-1) /[q r-(p-1)(s+1)]}
\end{aligned}
$$

and $u_{\epsilon, a}$ is the minimal energy solution of (1.4) given in Theorem A.

In analogy to Theorem $\mathrm{A}$, we also call $\left(A_{\epsilon, a}, \xi_{\epsilon, a}\right)$ a nearboundary spike if $0<a<a(N, p)$ and an interior spike if $a>a(N, p)$. 

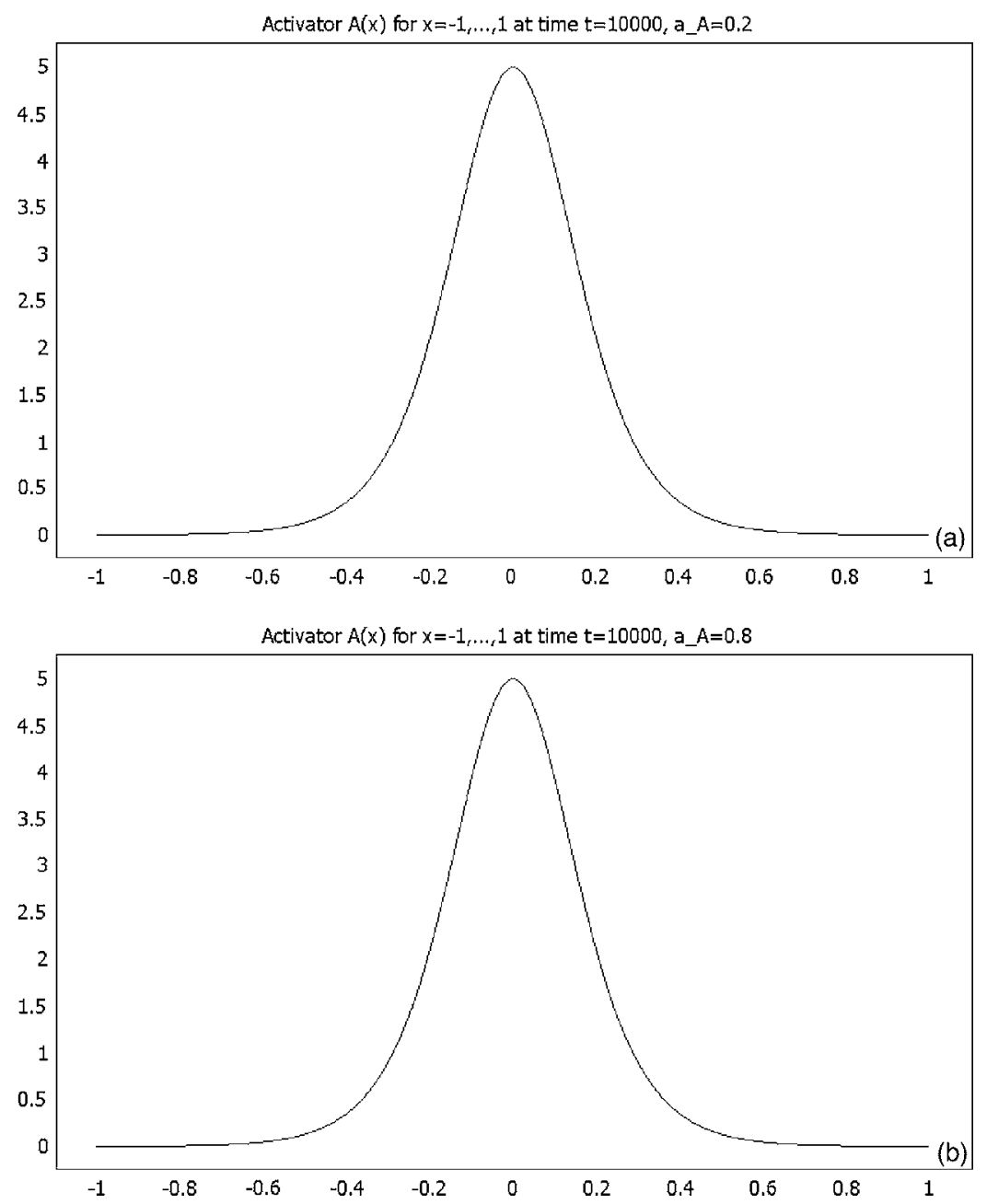

FIG. 2. Interior-boundary spike for variable constant $a_{A}$ in the Robin boundary. We have chosen $a_{A}=0.2,0.8$. It is numerically stable (final state is shown for $t$ $=10000)$.
For the Neumann boundary condition, a stability result has been obtained in Ref. 13 for

$$
r=2 \quad \text { and } 1<p<1+\frac{4}{N}
$$

or

$$
r=p+1 \quad \text { and } 1<p<\left(\frac{N+2}{N-2}\right)_{+} .
$$

In this paper, for Robin boundary conditions, we can give an answer under similar conditions.

Our first result implies that if $a>a(N, p)$, then the interior spike is stable.

Theorem 1.2 (stability of the interior spike). Suppose that $a>a(N, p)$. Assume that either

$$
r=2 \text { and } 1<p<1+\frac{4}{N}
$$

or

$$
r=p+1 \quad \text { and } 1<p<\left(\frac{N+2}{N-2}\right)_{+} .
$$

Then there exists $\tau_{0}>0$ such that if $0<\epsilon \ll 1$ and $0 \leq \tau$ $<\tau_{0}$, the interior spike $\left(A_{\epsilon, a}, \xi_{\epsilon, a}\right)$ is a (linearly) stable steady state to the shadow system (1.3).
Our second theorem shows that if $N=1$, i.e., if $\Omega$ is an interval, then in particular for all $1<p \leq 3$ and $0<a<1$ the near-boundary spike is stable.

Theorem 1.3. (stability of the near-boundary spike). Suppose that

$$
N=1 \text { and } 0<a<1 .
$$

Assume that either

$$
r=2 \text { and } 1<p \leq 3
$$

or

$$
r=p+1 \text { and } 1<p<\infty .
$$

Then there exists a $\tau_{0}>0$ such that if $0<\epsilon \ll 1$ and $0 \leq \tau$ $<\tau_{0}$, the near-boundary spike $\left(A_{\epsilon, a}, \xi_{\epsilon, a}\right)$ is a (linearly) stable steady state to the shadow system (1.3).

Our third and last theorem shows that the near-boundary spike may become unstable if the exponent $p$ is increased beyond 3 .

Theorem 1.4 (instability of the near-boundary spike). Suppose that (1.11) holds. Assume that $r=2$ and $p>3$. Then there exists $a_{0}>0$ and $\mu_{0}>0$ such that if

$$
a_{0}<a<1 \quad \text { and } 1<\mu:=\frac{2 q}{(p-1)(s+1)}<\mu_{0}
$$

then for $0<\epsilon \ll 1$ and all $\tau \geq 0$ the near-boundary spike 

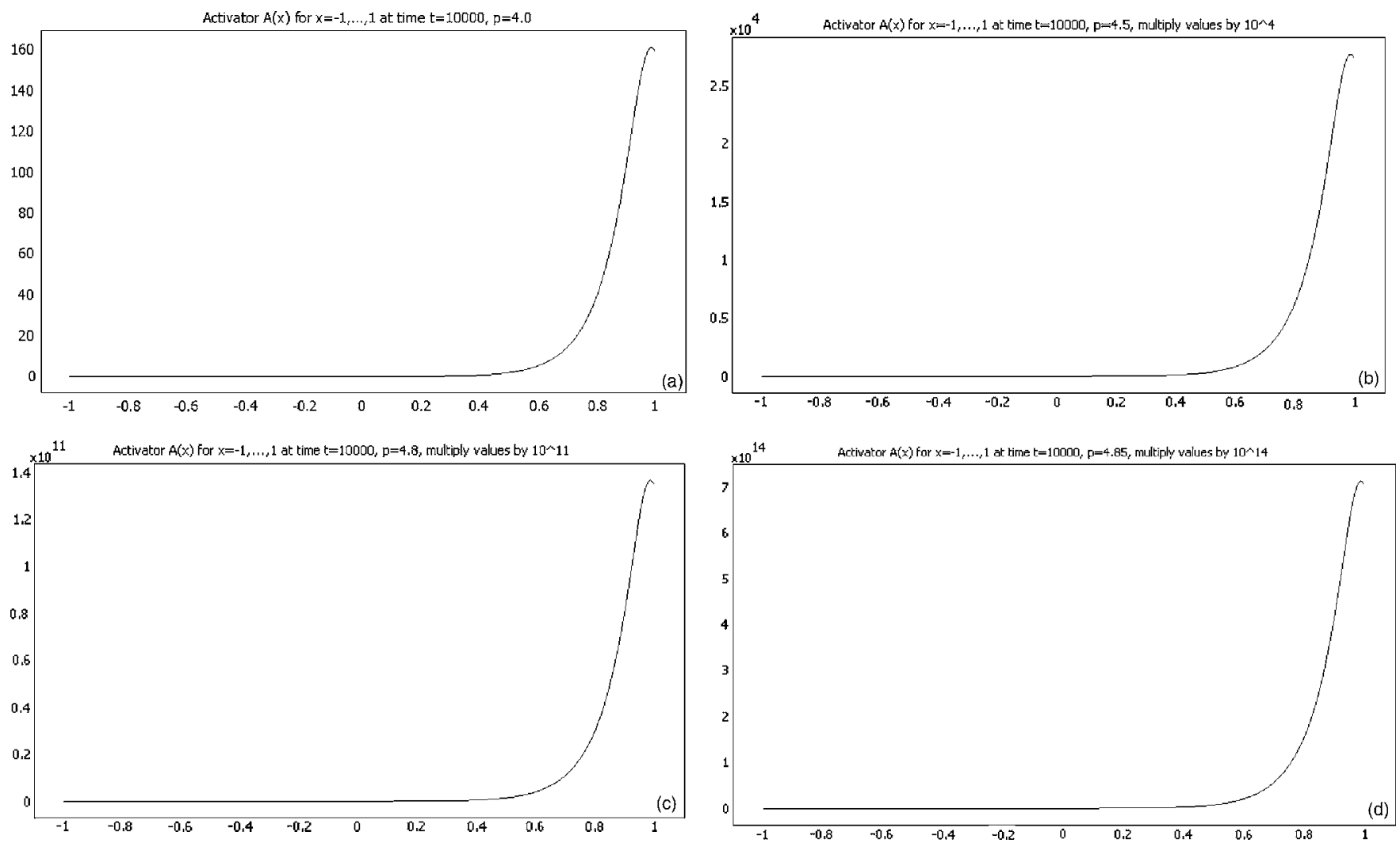

FIG. 3. Stable near-boundary spike. We choose constants $a_{A}=0.8, q=2, r=2, s=0$, and varying $p$. For $p=4.0,4.5,4.8$, and 4.85 , the near-boundary spike is shown. It is numerically stable (final state is shown for $t=10000$ ).

$\left(A_{\epsilon, a}, \xi_{\epsilon, a}\right)$ is an unstable steady state to the shadow system (1.3).

Remark 1.5. (1) The phenomenon described in Theorem 1.4 is new and unexpected. The proof shows that this instability is connected to an eigenvalue of order $O(1)$ as $\epsilon \rightarrow 0$. It is important to note that for $N=1$ and the Neumann boundary condition, the minimal energy solution analogous to Theorem A, which is a boundary spike, is stable for all p,q,s such that

$$
1<\mu:=\frac{2 q}{(p-1)(s+1)} \text { and } 1<p<5,
$$

see Ref. 14. This means that the instability given in Theorem 1.3 only arises for the Robin boundary condition and not for the Neumann boundary condition.

In some sense, for the Robin boundary condition the threshold for the instability that for Neumann boundary conditions occurs only for $p>5$ is shifted to the range $3<p$ $\leq 5$. The threshold $\mu=\mu_{0}$ corresponds to a Hopf bifurcation.

(2) Note that for the instability in Theorem 1.3 to occur, we assume that both the constants $a<1$ and $\mu:=\frac{q r}{(p-1)(s+1)}$ $>1$ are each sufficiently close to 1 .

(3) Note that under the conditions (1.12) a proof similar to the one for Theorem 1.1 shows that the interior spike is unstable due to an exponentially small eigenvalue (this proof is omitted). On the other hand, by Theorem 1.3, the nearboundary spike is unstable as well, due to an $O(1)$ instability, compare part (1) of this remark. Thus we do not know about any stable spiky steady state and we conjecture that there are none. This behavior is similar to the case $\mu$ $:=\frac{q r}{(p-1)(s+1)}<1$ for the Neumann boundary condition; see Ref. 9. In all of these situations, due to the nonexistence of stable steady states, oscillations with large amplitudes frequently occur for the dynamical system. This effect will be shown in the simulations of the dynamical system in the final section of this paper (see Figs. 3 and 4).

Let us now outline the proof of Theorems 1.2-1.4 by highlighting our strategy and explaining how we tackle the main difficulties.

To study the stability of the steady state, we have to linearize (1.3) at (1.9). This results in the following eigenvalue problem:

$$
\begin{aligned}
& \epsilon^{2} \Delta \phi_{\epsilon}-\phi_{\epsilon}+p \frac{A_{\epsilon}^{p-1}}{\xi_{\epsilon}^{q}} \phi_{\epsilon}-q \frac{A_{\epsilon}^{p}}{\xi_{\epsilon}^{q+1}} \eta_{\epsilon}=\alpha_{\epsilon} \phi_{\epsilon}, \\
& \frac{r}{\tau|\Omega|} \int_{\Omega} \frac{A_{\epsilon}^{r-1} \phi_{\epsilon}}{\xi_{\epsilon}^{s}} d x-\frac{1+s}{\tau} \eta_{\epsilon}=\alpha_{\epsilon} \eta_{\epsilon},
\end{aligned}
$$

where $\left(\phi_{\epsilon}, \eta_{\epsilon}\right)$ in $H_{\mathrm{rob}}^{1}(\Omega) \times R$ and

$$
H_{\mathrm{rob}}^{1}(\Omega)=\left\{\phi \in H^{1}(\Omega): \epsilon \frac{\partial \phi}{\partial \nu}+a \phi=0 \quad \text { on } \partial \Omega\right\} .
$$

Using (1.9), it is easy to see that for $a_{H}=0$, the eigenvalues of problem (1.13) in $H_{r o b}^{1}(\Omega) \times R$ are the same as the eigenvalues of the eigenvalue problem 

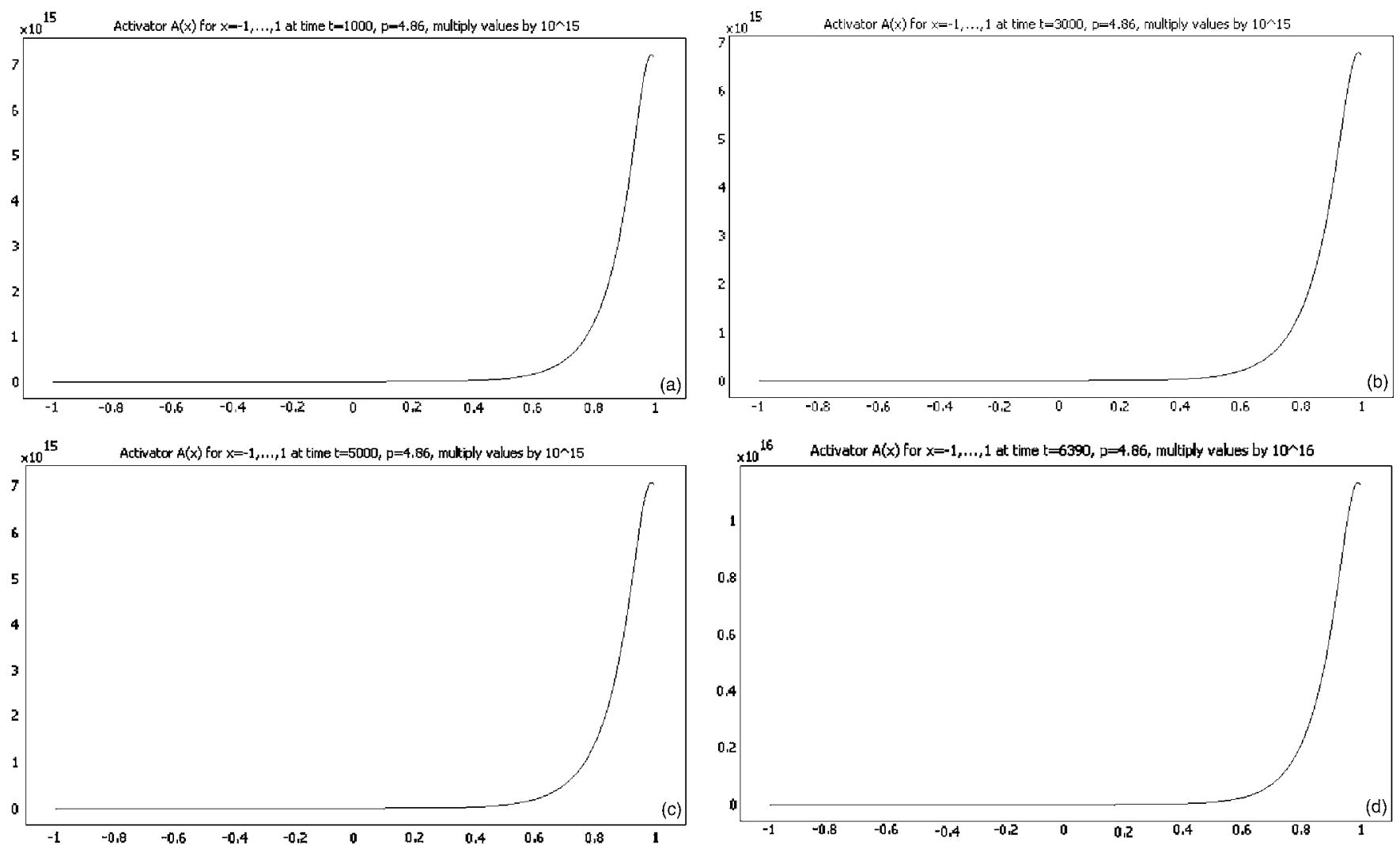

FIG. 4. Unstable near-boundary spike. We choose constants $a_{A}=0.8, q=2, r=2, s=0$, and $p=4.86$. The near-boundary spike is now numerically unstable. In the time evolution, the amplitude increases (shown for $t=1000,3000,5000$, and 6390).

$\epsilon^{2} \Delta \phi_{\epsilon}-\phi_{\epsilon}+p u_{\epsilon}^{p-1} \phi_{\epsilon}-\frac{q r}{s+1+\tau \alpha_{\epsilon}} \frac{\int_{\Omega} u_{\epsilon}^{r-1} \phi_{\epsilon} d x}{\int_{\Omega} u_{\epsilon}^{r} d x} u_{\epsilon}^{p}=\alpha_{\epsilon} \phi_{\epsilon}$,

$\phi_{\epsilon} \in H_{\mathrm{rob}}^{1}(\Omega)$.

When $N=1$ and $0<a<1$, we have $u_{\epsilon, a}(x) \sim w\left(\frac{x-x_{\epsilon}}{\epsilon}\right)$ $=w\left(\frac{x}{\epsilon}-\frac{x_{\epsilon}}{\epsilon}\right)=: w_{x_{\epsilon} \epsilon \epsilon}\left(\frac{x}{\epsilon}\right)$, where $w$ is the unique homoclinic solution of the second-order ODE,

$$
\begin{aligned}
& w^{\prime \prime}-w+w^{p}=0, \quad w>0, \quad w=w(|y|), \\
& w(y) \rightarrow 0 \text { as }|y| \rightarrow \infty .
\end{aligned}
$$

Further, by the Robin boundary condition $w^{\prime}=a w, \frac{x_{\epsilon}}{\epsilon} \rightarrow y_{0}$, where $y_{0}>0$ is determined by

$$
w^{\prime}\left(-y_{0}\right)=a w\left(-y_{0}\right)
$$

Now we use the explicit representation of the solution to the problem (1.15), which is given by

$$
w(y)=\left(\frac{p+1}{2} \cosh ^{-2} \frac{(p-1) y}{2}\right)^{1 /(p-1)} .
$$

We compute

$$
w^{\prime}(y)=-\tanh \frac{(p-1) y}{2} w(y) .
$$

This implies

$$
\frac{w^{\prime}\left(-y_{0}\right)}{w\left(-y_{0}\right)}=\tanh \frac{(p-1) y_{0}}{2}=a \text {. }
$$

So, if $0<a<1, y_{0}$ can explicitly be expressed in terms of $a$ as

$$
y_{0}=\frac{2}{p-1} \operatorname{artanh} a
$$

and we get

$$
w\left(y_{0}\right)=\left(\frac{(p+1)\left(1-a^{2}\right)}{2}\right)^{1 /(p-1)} .
$$

Let $\alpha_{\epsilon}$ be an eigenvalue of (1.14). Then the following Lemma holds.

Lemma A. (1) For $a>a(N, p)$ we have $\alpha_{\epsilon}=o(1)$ as $\epsilon$ $\rightarrow 0$ if and only if $\alpha_{\epsilon}=[1+o(1)] \tau_{j}^{\epsilon}$ for some $j=1, \ldots, N$, where $\tau_{j}^{\epsilon}$ is given in Theorem 3.4 below (interior spike case).

For $N=1$ and $a<a(N, p)$ there are no eigenvalues $\alpha_{\epsilon}$ $=o(1)$ (near-boundary spike case).

(2) If $\alpha_{\epsilon} \rightarrow \alpha_{0} \neq 0$, then all possible $\alpha_{0}$ are given by the eigenvalues of the following eigenvalue problem:

$\Delta \phi-\phi+p w_{y_{0}}^{p-1} \phi-\frac{q r}{s+1+\tau \alpha_{0}} \frac{\int_{0}^{\infty} w_{y_{0}}^{r-1} \phi}{\int_{0}^{\infty} w_{y_{0}}^{r}} w_{y_{0}}^{p}=\alpha_{0} \phi$,

where (i) for $a>a(N, P)$ we have $w_{y_{0}}=w, \phi \in H^{1}(R)$ (interior spike case) and (ii) for $N=1$ and $a<a(1, P)=1$ we have $w_{y_{0}}=w\left(y-y_{0}\right)$, where $y_{0}$ is given by the unique solution of 
$w^{\prime}\left(y_{0}\right)+a w\left(y_{0}\right)=0$, and $\phi \in H_{\mathrm{rob}}^{1}\left(R^{+}\right)$(near-boundary spike case).

Proof. When $a>a(N, p)$, the proof of part (1) in Lemma A for the Robin boundary condition is similar to that in Ref. 13 for the Neumann boundary condition. In both cases, because interior spikes are considered that have exponential decay with respect to the spatial variable, one has to expand the solution to exponential order. There is, however, a major difference in the stability properties. Whereas for the Neumann boundary condition interior spikes are unstable, they are stable for the Robin boundary condition. This difference comes from the fact that the expression $\varphi_{\epsilon, P}\left(P_{\epsilon}\right)$, which plays a major role in the proof [defined in Eq. (3.3)], has different signs for the Neumann and Robin boundary conditions, respectively.

When $N=1$ and $a<1$, the proof of part (1) in Lemma A for the Robin boundary condition is similar to that in Ref. 14 for a boundary spike with the Neumann boundary condition: In both cases, there are no small eigenvalues $\alpha_{\epsilon}=o(1)$.

The proof of (2) follows by a standard limiting process coupled with an argument of Dancer. ${ }^{15}$

Notice that the eigenvalue problem in part (2) of Lemma A (near-boundary spike case) is a half-line nonlocal eigenvalue problem NLEP with a Robin boundary condition. This is a new type of NLEP that to the best of our knowledge has not been studied in the literature before. We will prove results on its spectral and stability properties in the next section. For the study on NLEP, we refer to Refs. 24-30

From now on we assume that $\tau=0$. By a regular perturbation argument, the results also hold for the case of $\tau$ being sufficiently small.

\section{STUDY OF THE NLEP:}

\section{PROOF OF THEOREMS 1.2 AND 1.3}

In this section, we study the NLEP,

$$
\begin{aligned}
& \phi^{\prime \prime}-\phi+p w_{y_{0}}^{p-1} \phi-\frac{q r}{s+1} \frac{\int_{0}^{\infty} w_{y_{0}}^{r-1} \phi d y}{\int_{0}^{\infty} w_{y_{0}}^{r} d y} w_{y_{0}}^{p}=\lambda \phi, \\
& \phi \in H_{\mathrm{rob}}^{1}\left(R^{+}\right),
\end{aligned}
$$

where $w_{y_{0}}(y)=w\left(y-y_{0}\right)$ for some $y_{0}>0$, and $w$ satisfies (1.15). Let

$$
L_{0} \phi:=\phi^{\prime \prime}-\phi+p w_{y_{0}}^{p-1} \phi, \quad \phi \in H_{\mathrm{rob}}^{1}\left(R^{+}\right) .
$$

We set

$$
L \phi:=L_{0} \phi-\mu(p-1) \frac{\int_{0}^{\infty} w_{y_{0}}^{r-1} \phi d y}{\int_{0}^{\infty} w_{y_{0}}^{r} d y} w_{y_{0}}^{p}, \quad \phi \in H_{\mathrm{rob}}^{1}\left(R^{+}\right),
$$

where

$$
\mu=\frac{q r}{(s+1)(p-1)}>1 .
$$

We first prove the following Lemma.

Lemma 2.1. Let $\phi \in H_{\mathrm{rob}}^{1}\left(R^{+}\right)$satisfy

$$
\phi^{\prime \prime}-\phi+p w_{y_{0}}^{p-1} \phi=0, \quad\|\phi\|_{H^{1}\left(R^{+}\right)}=1 .
$$

Then $\phi \equiv 0$.
Proof. Recall that the Robin boundary condition gives

$$
a=\frac{w_{y_{0}}^{\prime}(0)}{w_{y_{0}}(0)}
$$

and by (1.15) $w_{y_{0}}$ satisfies

$$
w_{y_{0}}^{\prime \prime}=w_{y_{0}}-w_{y_{0}}^{p}, \quad\left(w_{y_{0}}^{\prime}\right)^{2}=w_{y_{0}}^{2}-\frac{2}{p+1} w_{y_{0}}^{p+1} .
$$

We multiply (2.2) by $w_{y_{0}}^{\prime}$ and integrate. After integration by parts, using (2.4) and the Robin boundary condition for $\phi$, we get

$$
0=\phi^{\prime}(0) w_{y_{0}}^{\prime}(0)-\phi(0) w_{y_{0}}^{\prime \prime}(0)=\phi(0)\left[a w_{y_{0}}^{\prime}(0)-w_{y_{0}}^{\prime \prime}(0)\right] \text {. }
$$

By (2.3) and (2.4) it follows that

$$
\begin{aligned}
a w_{y_{0}}^{\prime}(0)-w_{y_{0}}^{\prime \prime}(0) & =\frac{\left[w_{y_{0}}^{\prime}(0)\right]^{2}-w_{y_{0}}(0) w_{y_{0}}^{\prime \prime}(0)}{w_{y_{0}}(0)} \\
& =\frac{\left[w_{y_{0}}^{\prime}(0)\right]^{2}-\left[w_{y_{0}}(0)\right]^{2}+\left[w_{y_{0}}(0)\right]^{p+1}}{w_{y_{0}}(0)} \\
& =\frac{p-1}{p+1} w_{y_{0}}^{p}>0 .
\end{aligned}
$$

Thus from (2.5) we have

$$
\phi(0)=0
$$

and finally we get $\phi^{\prime}(0)=0$ by the Robin boundary condition. By the uniqueness properties of ODEs, we conclude that $\phi(y) \equiv 0$ on $R^{+}$. The lemma is proved.

Lemma 2.1 implies, using the Fredholm Alternative, that the operator $L_{0}$, defined on $H_{\text {rob }}^{1}\left(R^{+}\right)$, is invertible.

Since

$$
L_{0} w_{y_{0}}=(p-1) w_{y_{0}}^{p}, \quad w_{y_{0}}^{\prime}(0)-a w_{y_{0}}(0)=0
$$

we have

$$
L_{0}^{-1}\left(w_{y_{0}}^{p}\right)=\frac{1}{p-1} w_{y_{0}} .
$$

Another simple calculation shows that

$$
L_{0}\left(\frac{1}{p-1} w_{y_{0}}+\frac{1}{2} y w_{y_{0}}^{\prime}\right)=w_{y_{0}},
$$

but note that $\frac{1}{p-1} w_{y_{0}}+\frac{1}{2} y w_{x_{0}}^{\prime}$ does not satisfy the Robin boundary condition. Thus, since $\frac{1}{p-1} w_{y_{0}}+\frac{1}{2} y w_{y_{0}}^{\prime} \notin H_{\mathrm{rob}}^{1}\left(R^{+}\right)$, we do not have $L_{0}^{-1}\left(w_{y_{0}}\right)=\frac{1}{p-1} w_{y_{0}}+\frac{1}{2} y w_{y_{0}}^{\prime}$. To overcome this difficulty and determine $L_{0}^{-1}\left(w_{y_{0}}\right)$, we prove the following lemma.

Lemma 2.2. We have for $a \neq 1$ that

$$
L_{0}^{-1}\left(w_{y_{0}}\right)=\frac{1}{p-1} w_{y_{0}}+\frac{1}{2} y w_{y_{0}}^{\prime}+A w_{y_{0}}^{\prime},
$$

where

$$
A=\frac{a}{(p-1)\left(1-a^{2}\right)} .
$$


Proof. We need to choose $A$ such that

$$
A\left[w_{y_{0}}^{\prime \prime}(0)-a w_{y_{0}}^{\prime}(0)\right]+\frac{1}{2} w_{y_{0}}^{\prime}(0)=0 .
$$

Using (2.6), we get

$$
A=\frac{p+1}{2(p-1)} w_{y_{0}}^{\prime}(0) w_{y_{0}}^{-p}(0) \text {. }
$$

Inserting (1.18) into (2.10), we get

$$
A=\frac{p+1}{2(p-1)}\left(\frac{p+1}{2}\right)^{-1} \frac{a}{1-a^{2}}=\frac{a}{(p-1)\left(1-a^{2}\right)},
$$

which proves the lemma.

Remark 2.3 (1) In the multidimensional case it is unclear how to find an equivalent to the correction term given in Lemma 2.2 (and starting with the factor A).

(2) The extra term $A w_{y_{0}}^{\prime}$ in Lemma 2.2 only appears for Robin boundary conditions and is not present for the Neumann boundary condition. As we will see, the presence of this term under some extra conditions can lead to the destabilization of the near-boundary spike.

(3) Note that $A \rightarrow \infty$ as $a \rightarrow 1$ and $A \rightarrow 0$ as $a \rightarrow 0$. The first limit will play a major role for the rest of the paper. The second limit is in agreement with intuition since in the limit $a \rightarrow 0$ the near-boundary spike for the Robin boundary condition approaches the boundary spike for the Neumann boundary condition, where this term does not appear.

We now compute the sign of the expression

$$
\rho\left(y_{0}\right):=\int_{0}^{\infty} w_{y_{0}} L_{0}^{-1}\left(w_{y_{0}}\right) d y
$$

which will play the crucial role in the stability analysis of the near-boundary spike.

From Lemma 2.2, we have

$$
\begin{aligned}
\rho\left(y_{0}\right)= & \int_{0}^{\infty} w_{y_{0}} L_{0}^{-1}\left(w_{y_{0}}\right) d y \\
= & \frac{1}{p-1} \int_{0}^{\infty} w_{y_{0}}^{2} d y+\frac{1}{2} \int_{0}^{\infty} y w_{y_{0}} w_{y_{0}}^{\prime} d y+A \int_{0}^{\infty} w_{y_{0}} w_{y_{0}}^{\prime} d y \\
= & \left(\frac{1}{p-1}-\frac{1}{4}\right) \int_{0}^{\infty} w_{y_{0}}^{2} d y-\frac{A}{2} w_{y_{0}}^{2}(0) \\
= & \left(\frac{1}{p-1}-\frac{1}{4}\right) \int_{0}^{\infty} w_{y_{0}}^{2} d y \\
& -\frac{a}{2(p-1)\left(1-a^{2}\right)}\left(\frac{(p+1)\left(1-a^{2}\right)}{2}\right)^{2 /(p-1)} \\
= & \left(\frac{1}{p-1}-\frac{1}{4}\right) \int_{-y_{0}}^{\infty} w^{2} d y \\
& -\frac{(p+1)^{2 /(p-1)} a}{2^{(p+1) /(p-1)}(p-1)\left(1-a^{2}\right)^{(p-3) /(p-1)}} .
\end{aligned}
$$

Let us differentiate $\rho\left(y_{0}\right)$ with respect to $y_{0}$. Using (2.10), we get

$$
\begin{aligned}
\rho^{\prime}\left(y_{0}\right)= & \frac{5-p}{4(p-1)} w_{y_{0}}^{2}(0) \\
& +\frac{p+1}{4(p-1)}\left\{w_{y_{0}}^{2-p}(0) w_{y_{0}}^{\prime \prime}(0)\right. \\
& \left.+(2-p) w_{y_{0}}^{1-p}(0)\left[w_{y_{0}}^{\prime}(0)\right]^{2}\right\} \\
= & \frac{5-p}{4(p-1)} w_{y_{0}}^{2}(0)+\frac{p+1}{4(p-1)}\left[\left(w_{y_{0}}-w_{y_{0}}^{p}\right) w_{y_{0}}^{2-p}\right. \\
& \left.+(2-p) w_{y_{0}}^{1-p}\left(w_{y_{0}}^{2}-\frac{2}{p+1} w_{y_{0}}^{p+1}\right)\right](0) \\
= & \frac{(p+1)(3-p)}{4(p-1)} w_{y_{0}}^{3-p}(0)
\end{aligned}
$$

by (2.4). We arrive at the following important proposition.

Proposition 2.4. Suppose that $1<p \leq 3$. Then

$$
\int_{0}^{\infty} w_{y_{0}} L_{0}^{-1}\left(w_{y_{0}}\right) d y>0 .
$$

Proof. For $1<p \leq 3$, we get from (2.11) for $y_{0}=0$ (and so also $a=0$ ) that

$$
\rho(0)=\left(\frac{1}{p-1}-\frac{1}{4}\right) \int_{0}^{\infty} w_{y_{0}}^{2} d y>0 .
$$

By (2.12) we compute $\rho^{\prime}\left(y_{0}\right) \geq 0$ for all $y_{0} \in(0, \infty)$ and therefore $\rho\left(y_{0}\right) \geq 0$ for all $y_{0} \in[0, \infty)$.

We now show that for $p>3$, in contrast to Proposition 2.4, the integral $\int_{0}^{\infty} w_{y_{0}} L_{0}^{-1}\left(w_{y_{0}}\right) d y$ may be negative.

Proposition 2.5. Suppose that $p>3$. Then for

$\frac{5-p}{p-1} \int_{-(2 p-1) \operatorname{artanh} a}^{\infty} w^{2} d y<\frac{2^{(p-3) /(p-1)}(p+1)^{2 /(p-1)} a}{(p-1)\left(1-a^{2}\right)^{(p-3) /(p-1)}}$

it follows that $\int_{0}^{\infty} w_{y_{0}} L_{0}^{-1}\left(w_{y_{0}}\right) d y$ is negative. There exists some constant $a_{0}(p)<1$ such for $a_{0}(p)<a<1$, condition (2.14) holds.

Before proceeding to the proof, it is enlightening to consider the restriction posed by condition (2.14). This is done in the following remarks.

Remark 2.6. (1) For $p=5$, (2.14) is satisfied for all 0 $<a<1$ as 1.h.s. $=0$ and it poses no restriction at all. This implies that $a_{0}(p) \rightarrow 0$ as $p \rightarrow 5^{-}$.

(2) The solution set of (2.14) within $0<a<1$ is empty if $3<p<p_{0}$ for some $p_{0}>3$ as for $p=3$ r.h.s. $=2 a$ and 1.h.s. $>2$.

(3) We now consider the asymptotic behavior of Eq. (2.14) in the most interesting range $p \rightarrow 5^{-}$. In leading order we get

$$
\frac{\pi}{4}(5-p)<a+O\left(a^{2}\right)+O\left[(5-p)^{2}\right] .
$$

(4) For $p$ close to 5 (and possibly for the whole range of $3<p<5)$ the threshold $a_{0}(p)$ given by (2.14) is uniquely defined and $a_{0}(p)$ is monotone decreasing in $p$.

Proof. Condition (2.14) follows immediately from (2.11). The left-hand side of (2.14) is positive and remains bounded for all $a \in(0,1)$. The right-hand side of (2.14) tends 
to 0 as $a \rightarrow 0^{+}$and to $+\infty$ as $a \rightarrow 1^{-}$. By continuity, there exists some $a_{0}(p) \in(0,1)$ such that $(2.14)$ is true for $a_{0}(p)$ $<a<1$.

Next we need the following.

Lemma 2.7. The first eigenvalue of $L_{0}$, which we call $\mu_{1}$, is positive. The second eigenvalue of $L_{0}$ is negative.

Proof. Let

$$
Q[u]=\frac{\int_{0}^{\infty}\left[\left(u^{\prime}\right)^{2}+u^{2}\right] d y+a u^{2}(0)}{\left(\int_{0}^{\infty} u^{p+1} d y\right)^{2 /(p+1)}} .
$$

Then $w_{y_{0}}$ up to a scaling factor is the unique minimizer of $Q[u]$ in $H_{\text {rob }}^{1}\left(R^{+}\right)$.

Similar to the proof of Theorem 2.1 of Ref. 16, we see that the second eigenvalue of $L_{0}$ is nonpositive, and hence is negative since by Lemma 2.1 the kernel is trivial.

Now, to study the case $r=2$, we introduce a new operator,

$$
\begin{aligned}
L_{1} \phi:= & L_{0} \phi-(p-1) \frac{\int_{0}^{\infty} w_{y_{0}} \phi d y}{\int_{0}^{\infty} w_{y_{0}}^{2} d y} w_{y_{0}}^{p}-(p-1) \frac{\int_{0}^{\infty} w_{y_{0}}^{p} \phi d y}{\int_{0}^{\infty} w_{y_{0}}^{2} d y} w_{y_{0}} \\
& +(p-1) \frac{\int_{0}^{\infty} w_{y_{0}}^{p+1} d y \int_{0}^{\infty} w_{y_{0}} \phi d y}{\left(\int_{0}^{\infty} w_{y_{0}}^{2} d y\right)^{2}} w_{y_{0}},
\end{aligned}
$$

which is defined for all $\phi \in H_{\mathrm{rob}}^{1}\left(R^{+}\right)$.

Then we have

Lemma 2.8. (1) The operator $L_{1}$ is self-adjoint and the kernel of $L_{1}$ (denoted by $\left.X_{1}\right)$ is given by $\operatorname{span}\left\{w_{y_{0}}\right\}$.

(2) There exists a positive constant $c_{0}>0$ such that

$$
\begin{aligned}
L_{1}(\phi, \phi):= & \int_{0}^{\infty}\left[\left(\phi^{\prime}\right)^{2}+\phi^{2}-p w_{y_{0}}^{p-1} \phi^{2}\right] d y \\
& +\frac{2(p-1) \int_{0}^{\infty} w_{y_{0}} \phi d y \int_{0}^{\infty} w_{y_{0}}^{p} \phi d y}{\int_{0}^{\infty} w_{y_{0}}^{2} d y} \\
& -(p-1) \frac{\int_{0}^{\infty} w_{y_{0}}^{p+1} d y}{\left(\int_{0}^{\infty} w_{y_{0}}^{2} d y\right)^{2}}\left(\int_{0}^{\infty} w_{y_{0}} \phi d y\right)^{2} \\
\geq & c_{0} d_{L^{2}\left(R^{+}\right)}^{2}\left(\phi, X_{1}\right)
\end{aligned}
$$

for all $\phi \in H_{\mathrm{rob}}^{1}(0, \infty)$, where $d_{L^{2}(0, \infty)}$ denotes the distance in the $L^{2}$ norm.

Proof. By definition (2.15), it is an elementary calculation to show that $\left(L_{1} \phi, \psi\right)_{L^{2}(0, \infty)}=\left(L_{1} \psi, \phi\right)_{L^{2}(0, \infty)}$ for all $\phi, \psi$ $\in H^{1}(0, \infty)$, which implies that the operator $L_{1}$ is selfadjoint.

Next we compute the kernel of $L_{1}$. It is easy to see that $w_{y_{0}} \in \operatorname{kernel}\left(L_{1}\right)$. On the other hand, if $\phi \in \operatorname{kernel}\left(L_{1}\right)$, then

$$
\begin{aligned}
L_{0} \phi= & c_{1}(\phi) w_{y_{0}}+c_{2}(\phi) w_{y_{0}}^{p} \\
= & c_{1}(\phi) L_{0}\left(\frac{1}{p-1} w_{y_{0}}+\frac{1}{2} y w_{y_{0}}^{\prime}+A w_{y_{0}}^{\prime}\right) \\
& +c_{2}(\phi) L_{0}\left(\frac{1}{p-1} w_{y_{0}}\right)
\end{aligned}
$$

by Lemma 2.2, where

$$
\begin{aligned}
& c_{1}(\phi)=(p-1) \frac{\int_{0}^{\infty} w_{y_{0}}^{p} \phi d y}{\int_{0}^{\infty} w_{y_{0}}^{2} d y}-(p-1) \frac{\int_{0}^{\infty} w_{y_{0}}^{p+1} d y \int_{0}^{\infty} w_{y_{0}} \phi d y}{\left(\int_{0}^{\infty} w_{y_{0}}^{2} d y\right)^{2}}, \\
& c_{2}(\phi)=(p-1) \frac{\int_{0}^{\infty} w_{y_{0}} \phi d y}{\int_{0}^{\infty} w_{y_{0}}^{2} d y} .
\end{aligned}
$$

Hence

$$
\begin{aligned}
\phi & =c_{1}(\phi) L_{0}^{-1}\left(w_{y_{0}}\right)+c_{2}(\phi) L_{0}^{-1}\left(w_{y_{0}}^{p}\right) \\
& =c_{1}(\phi) L_{0}^{-1}\left(w_{y_{0}}\right)+\frac{1}{p-1} c_{2}(\phi) w_{y_{0}} .
\end{aligned}
$$

Note that by (2.16)

$$
\begin{aligned}
c_{1}(\phi)= & c_{1}(\phi)\left[(p-1) \frac{\int_{0}^{\infty} w_{y_{0}}^{p} L_{0}^{-1}\left(w_{y_{0}}\right) d y}{\int_{0}^{\infty} w_{y_{0}}^{2} d y}\right. \\
& \left.-(p-1) \frac{\int_{0}^{\infty} w_{y_{0}}^{p+1} d y \int_{0}^{\infty} w_{y_{0}} L_{0}^{-1}\left(w_{y_{0}}\right) d y}{\left(\int_{0}^{\infty} w_{y_{0}}^{2} d y\right)^{2}}\right] \\
= & c_{1}(\phi)\left[1-(p-1) \frac{\int_{0}^{\infty} w_{y_{0}}^{p+1} d y \int_{0}^{\infty} w_{y_{0}} L_{0}^{-1}\left(w_{y_{0}}\right) d y}{\left(\int_{0}^{\infty} w_{y_{0}}^{2} d y\right)^{2}}\right] .
\end{aligned}
$$

This implies that $c_{1}(\phi)=0$. By (2.16) and Lemma 2.1, this proves (1).

It remains to prove (2). Suppose (2) is not true, then by (1) there exists $(\alpha, \phi)$ such that (i) $\alpha>0$, (ii) $\phi \perp w_{y_{0}}$, and (iii) $L_{1} \phi=\alpha \phi$.

We show that this is impossible. From (ii) and (iii), we have

$$
\left(L_{0}-\alpha\right) \phi=(p-1) \frac{\int_{0}^{\infty} w_{y_{0}}^{p} \phi d y}{\int_{0}^{\infty} w_{y_{0}}^{2} d y} w_{y_{0}} .
$$

We first claim that $\int_{0}^{\infty} w_{y_{0}}^{p} \phi d y \neq 0$. In fact, if $\int_{0}^{\infty} w_{y_{0}}^{p} \phi d y=0$, then $\alpha>0$ is the first (principal) eigenvalue of $L_{0}$. By Proposition 2.7, $\alpha=\mu_{1}$ and $\phi$ has a constant sign. This contradicts (ii).

Therefore, we must have $\int_{0}^{\infty} w_{y_{0}}^{p} \phi d y \neq 0$ Hence $\alpha \neq \mu_{1}$ and $L_{0}-\alpha$ is invertible. So (2.17) implies

$$
\phi=(p-1) \frac{\int_{0}^{\infty} w_{y_{0}}^{p} \phi d y}{\int_{0}^{\infty} w_{y_{0}}^{2} d y}\left(L_{0}-\alpha\right)^{-1} w_{y_{0}} .
$$

Thus

$$
\begin{aligned}
& \int_{0}^{\infty} w_{y_{0}}^{p} \phi d y=(p-1) \frac{\int_{0}^{\infty} w_{y_{0}}^{p} \phi d y}{\int_{0}^{\infty} w_{y_{0}}^{2} d y} \int_{0}^{\infty}\left[\left(L_{0}-\alpha\right)^{-1} w_{y_{0}}\right] w_{y_{0}}^{p} d y, \\
& \int_{0}^{\infty} w_{y_{0}}^{2} d y=(p-1) \int_{0}^{\infty}\left[\left(L_{0}-\alpha\right)^{-1} w_{y_{0}}\right] w_{y_{0}}^{p} d y, \\
& \int_{0}^{\infty} w_{y_{0}}^{2} d y=\int_{0}^{\infty}\left[\left(L_{0}-\alpha\right)^{-1} w_{y_{0}}\right]\left[\left(L_{0}-\alpha\right) w_{y_{0}}+\alpha w_{y_{0}}\right] d y,
\end{aligned}
$$




$$
0=\int_{0}^{\infty}\left[\left(L_{0}-\alpha\right)^{-1} w_{y_{0}}\right] w_{y_{0}} d y .
$$

Let $\quad h_{1}(\alpha)=\int_{0}^{\infty}\left[\left(L_{0}-\alpha\right)^{-1} w_{y_{0}}\right] w_{y_{0}} d y$. Then $\quad h_{1}(0)$ $=\int_{0}^{\infty}\left(L_{0}^{-1} w_{y_{0}}\right) w_{y_{0}} d y=\rho_{0}(\alpha)>0$ by Proposition 2.4. Moreover $h_{1}^{\prime}(\alpha)=\int_{0}^{\infty}\left[\left(L_{0}-\alpha\right)^{-2} w_{y_{0}}\right] w_{y_{0}}=\int_{0}^{\infty}\left[\left(L_{0}-\alpha\right)^{-1} w_{y_{0}}\right]^{2} d y>0$. This implies $h_{1}(\alpha)>0$ for all $\alpha \in\left(0, \mu_{1}\right)$. Clearly, since $\lim _{\alpha \rightarrow+\infty} h_{1}(\alpha)=0^{-}$, we also have $h_{1}(\alpha)<0$ for $\alpha \in\left(\mu_{1}, \infty\right)$.

This is a contradiction to (2.18), and completes the proof.

First we have the following theorem about (in)stability of a near-boundary spike in the case of the Robin boundary condition including the exponents $r=2,1<p \leq 3$, which is similar to the results for an interior or a boundary spike in the case of the Neumann boundary condition:

Theorem 2.9. Suppose $0<a<1$. If

$$
r=2 \text { and } 1<p \leq 3
$$

or if

$$
r=p+1 \text { and } 1<p<\infty,
$$

then the following NLEP

$\phi^{\prime \prime}-\phi+p w_{y_{0}}^{p-1} \phi-\mu(p-1) \frac{\int_{0}^{\infty} w_{y_{0}}^{r-1} \phi d y}{\int_{0}^{\infty} w_{y_{0}}^{r} d y} w_{y_{0}}^{p}=\lambda \phi$,

$\phi \in H_{\mathrm{rob}}^{1}\left(R^{+}\right)$,

for $\mu>1$ has only stable eigenvalues but for $\mu<1$ admits unstable eigenvalues.

In contrast, for the exponents $r=2, p>3$, we have the following instability result for the near-boundary spike in the case of Robin boundary condition:

\section{Theorem 2.10. If}

$r=2$ and $p>3$,

then there exist some $a_{0}(p) \in(0,1)$ and $\mu_{0}(a)>1$ such that for

$$
a_{0}<a<1
$$

and

$$
\mu<\mu_{0}(a)
$$

the NLEP (2.19) has a positive eigenvalue.

Remark 2.11 (1) The number $a_{0}$ can be chosen according to (2.14). See also Remark 2.6.

(2) It follows from continuity that $\mu_{0}(p) \rightarrow 1$ as a $\rightarrow a_{0}(p)$.

(3) At $\mu=\mu_{0}(p)$, Hopf bifurcation occurs. In fact, for $\mu$ large, the NLEP is stable (a rigorous proof of this will appear elsewhere). On the other hand, for $\mu<\mu_{0}(p), N L E P$ is unstable. Thus, as $\mu$ increases, the eigenvalues of NLEP will cross the imaginary axis. Since $\mu_{0}(p)>1$, eigenvalues cannot cross zero. Thus there exists a Hopf bifurcation. We remark that in the case of the Neumann boundary condition (i.e., $a=0)$, Ward and Wei have shown that when $p>5$, Hopf bifurcation can occur for some $\mu>1$. See the discussions in Sec. IV of Ref. 9.
To show the instability part in Theorems 2.9 and 2.10, we first prove the following result.

Theorem 2.12 (1) If $\mu<1$ and $r=2,1<p \leq 3$ or $r=p$ $+1,1<p<\infty$, the NLEP (2.19) has a positive eigenvalue.

(2) If $r=2$ and

$$
\int_{0}^{\infty} w_{y_{0}} L_{0}^{-1} w_{y_{0}} d y<0
$$

then under the condition (2.21) the NLEP (2.19) has a positive eigenvalue.

Proof. (1) Suppose $\mu<1$. We look for a positive eigenvalue $\alpha$ to (2.19) which is equivalent to

$\phi=\mu(p-1) \frac{\int_{0}^{\infty} w_{y_{0}}^{r-1} \phi d y}{\int_{0}^{\infty} w_{y_{0}}^{r} d y}\left(L_{0}-\alpha\right)^{-1} w_{y_{0}}^{p}, \quad 0<y<+\infty$,

$\phi^{\prime}(0)-a \phi(0)=0$.

Multiplying by $w_{y_{0}}^{r-1}$ and integrating, we get

$$
\int_{0}^{\infty} w_{y_{0}}^{r} d y=\mu(p-1) \int_{0}^{\infty}\left[\left(L_{0}-\alpha\right)^{-1} w_{y_{0}}^{p}\right] w_{y_{0}}^{r-1} d y .
$$

Using the identity

$$
(p-1)\left(L_{0}-\alpha\right)^{-1} w_{y_{0}}^{p-1}=w_{y_{0}}+\alpha\left(L_{0}-\alpha\right)^{-1} w_{y_{0}}
$$

we get

$\int_{0}^{\infty} w_{y_{0}}^{r} d y=\mu\left\{\int_{0}^{\infty} w_{y_{0}}^{r} d y+\alpha \int_{0}^{\infty}\left[\left(L_{0}-\alpha\right)^{-1} w_{y_{0}}\right] w_{y_{0}}^{r-1} d y\right\}$

which is equivalent to

$\frac{1}{\alpha}\left(\frac{1}{\mu}-1\right) \int_{0}^{\infty} w_{y_{0}}^{r} d y=\int_{0}^{\infty}\left[\left(L_{0}-\alpha\right)^{-1} w_{y_{0}}\right] w_{y_{0}}^{r-1} d y$.

If $r=2$ and $1<p \leq 3$, then by Proposition 2.4 the right-hand side of (2.22) is positive for $\alpha=0$.

If $r=p+1$ and $1<p<\infty$, then the right-hand side of (2.22) is positive for $\alpha=0$ since

$$
\int_{0}^{\infty}\left[L_{0}^{-1} w_{y_{0}}\right] w_{y_{0}}^{p} d y=\frac{1}{p-1} \int_{0}^{\infty} w_{y_{0}}^{2} d y>0 .
$$

Therefore, as $\alpha \rightarrow 0^{+}$, the left-hand side of (2.22) tends to $+\infty$ while the right-hand side tends to some positive number. As $\alpha \rightarrow \mu_{1}^{-}$, the left-hand side tends to some positive number while the right-hand side tends to $+\infty$. By continuity, there exists a solution to (2.22).

(2) If $r=2$, then (2.22) becomes

$$
\frac{1}{\alpha}\left(\frac{1}{\mu}-1\right) \int_{0}^{\infty} w_{y_{0}}^{2} d y=\int_{0}^{\infty}\left[\left(L_{0}-\alpha\right)^{-1} w_{y_{0}}\right] w_{y_{0}} d y .
$$

As $\alpha \rightarrow 0^{+}$, the left-hand side of (2.23) tends to $-\infty$ while the right-hand side tends to some negative number. As $\alpha$ $\rightarrow \mu_{1}^{-}$, the left-hand side tends to some negative number while the right-hand side tends to $-\infty$.

By continuity, there exists a solution to (2.23).

\section{Proof of Theorem 2.10:}

The proof of Theorem 2.10 is completed by combining Proposition 2.5 and part (2) of Theorem 2.12. 
Proof of Theorem 2.9:

The instability part of Theorem 2.9 is contained in part (1) of Theorem 2.12.

Now we prove the stability part of Theorem 2.9. We divide the proof into two cases. The outline follows Appendix F of Ref. 6:

Case 1. $r=2,1<p \leq 3$.

Case 2. $r=p+1,1<p<\infty$.

Let $\alpha_{0}=\alpha_{R}+i \alpha_{I}$ be an eigenvalue and $\phi=\phi_{R}+i \phi_{I}$ an eigenfunction of (2.19). Since $\alpha_{0} \neq 0$, we can choose $\phi \perp \operatorname{kernel}\left(L_{0}\right)$. Then we obtain the two equations

$$
\begin{aligned}
& L_{0} \phi_{R}-(p-1) \mu \frac{\int_{0}^{\infty} w_{y_{0}} \phi_{R} d y}{\int_{0}^{\infty} w_{y_{0}}^{2} d y} w_{y_{0}}^{p}=\alpha_{R} \phi_{R}-\alpha_{I} \phi_{I}, \\
& L_{0} \phi_{I}-(p-1) \mu \frac{\int_{0}^{\infty} w_{y_{0}} \phi_{I} d y}{\int_{0}^{\infty} w_{y_{0}}^{2} d y} w_{y_{0}}^{p}=\alpha_{R} \phi_{I}+\alpha_{I} \phi_{R} .
\end{aligned}
$$

Multiplying (2.24) by $\phi_{R}$ and (2.25) by $\phi_{I}$ and adding the two equations, we obtain

$$
\begin{aligned}
-\alpha_{R} \int_{0}^{\infty}\left(\phi_{R}^{2}+\phi_{I}^{2}\right) d y= & L_{1}\left(\phi_{R}, \phi_{R}\right)+L_{1}\left(\phi_{I}, \phi_{I}\right)+(p-1)(\mu-2) \frac{\int_{0}^{\infty} w_{y_{0}} \phi_{R} d y \int_{0}^{\infty} w_{y_{0}}^{p} \phi_{R} d y+\int_{0}^{\infty} w_{y_{0}} \phi_{I} d y \int_{0}^{\infty} w_{y_{0}}^{p} \phi_{I} d y}{\int_{0}^{\infty} w_{y_{0}}^{2} d y} \\
& +(p-1) \frac{\int_{0}^{\infty} w_{y_{0}}^{p+1} d y}{\left(\int_{0}^{\infty} w_{y_{0}}^{2}\right)^{2} d y}\left[\left(\int_{0}^{\infty} w_{y_{0}} \phi_{R} d y\right)^{2}+\left(\int_{0}^{\infty} w_{y_{0}} \phi_{I} d y\right)^{2}\right] .
\end{aligned}
$$

Multiplying (2.24) by $w_{y_{0}}$ and (2.25) by $w_{y_{0}}$, respectively, and integrating, we obtain

$$
\begin{aligned}
& (p-1) \int_{0}^{\infty} w_{y_{0}}^{p} \phi_{R} d y-(p-1) \mu \frac{\int_{0}^{\infty} w_{y_{0}} \phi_{R} d y}{\int_{0}^{\infty} w_{y_{0}}^{2} d y} \int_{0}^{\infty} w_{y_{0}}^{p+1} d y=\alpha_{R} \int_{0}^{\infty} w_{y_{0}} \phi_{R} d y-\alpha_{I} \int_{0}^{\infty} w_{y_{0}} \phi_{I} d y, \\
& (p-1) \int_{0}^{\infty} w_{y_{0}}^{p} \phi_{I} d y-(p-1) \mu \frac{\int_{0}^{\infty} w_{y_{0}} \phi_{I} d y}{\int_{0}^{\infty} w_{y_{0}}^{2} d y} \int_{0}^{\infty} w_{y_{0}}^{p+1} d y=\alpha_{R} \int_{0}^{\infty} w_{y_{0}} \phi_{I} d y+\alpha_{I} \int_{0}^{\infty} w_{y_{0}} \phi_{R} d y .
\end{aligned}
$$

Multiplying (2.26) by $\int_{0}^{\infty} w_{y_{0}} \phi_{R} d y$ and (2.27) by $\int_{0}^{\infty} w_{y_{0}} \phi_{I} d y$ and adding, we obtain

$$
\begin{aligned}
& (p-1) \int_{0}^{\infty} w_{y_{0}} \phi_{R} d y \int_{0}^{\infty} w_{y_{0}}^{p} \phi_{R} d y+(p-1) \int_{0}^{\infty} w_{y_{0}} \phi_{I} d y \int_{0}^{\infty} w_{y_{0}}^{p} \phi_{I} d y \\
& =\left(\alpha_{R}+(p-1) \mu \frac{\int_{0}^{\infty} w_{y_{0}}^{p+1} d y}{\int_{0}^{\infty} w_{y_{0}}^{2} d y}\right)\left[\left(\int_{0}^{\infty} w_{y_{0}} \phi_{R} d y\right)^{2}+\left(\int_{0}^{\infty} w_{y_{0}} \phi_{I} d y\right)^{2}\right] .
\end{aligned}
$$

Therefore, we have

$$
\begin{aligned}
-\alpha_{R} \int_{0}^{\infty}\left(\phi_{R}^{2}+\phi_{I}^{2}\right) d y= & L_{1}\left(\phi_{R}, \phi_{R}\right)+L_{1}\left(\phi_{I}, \phi_{I}\right)+(p-1)(\mu-2)\left(\frac{1}{p-1} \alpha_{R}+\mu \frac{\int_{0}^{\infty} w_{y_{0}}^{p+1} d y}{\int_{0}^{\infty} w_{y_{0}}^{2} d y}\right) \frac{\left(\int_{0}^{\infty} w_{y_{0}} \phi_{R} d y\right)^{2}+\left(\int_{0}^{\infty} w_{y_{0}} \phi_{I} d y\right)^{2}}{\int_{0}^{\infty} w_{y_{0}}^{2} d y} \\
& +(p-1) \frac{\int_{0}^{\infty} w_{y_{0}}^{p+1} d y}{\left(\int_{0}^{\infty} w_{y_{0}}^{2}\right)^{2}}\left[\left(\int_{0}^{\infty} w_{y_{0}} \phi_{R} d y\right)^{2}+\left(\int_{0}^{\infty} w_{y_{0}} \phi_{I} d y\right)^{2}\right]
\end{aligned}
$$

Set

$$
\begin{aligned}
& \phi_{R}=c_{R} w_{y_{0}}+\phi_{R}^{\perp}, \quad \phi_{R}^{\perp} \perp X_{1}, \\
& \phi_{I}=c_{I} w_{y_{0}}+\phi_{I}^{\perp}, \quad \phi_{I}^{\perp} \perp X_{1},
\end{aligned}
$$

where $X_{1}$ was defined in Lemma 2.8. Then

$$
\begin{aligned}
& \int_{0}^{\infty} w_{y_{0}} \phi_{R} d y=c_{R} \int_{0}^{\infty} w_{y_{0}}^{2} d y, \\
& \int_{0}^{\infty} w_{y_{0}} \phi_{I} d y=c_{I} \int_{0}^{\infty} w_{y_{0}}^{2} d y,
\end{aligned}
$$

$$
d_{L^{2}\left(R^{+}\right)}^{2}\left(\phi_{R}, X_{1}\right)=\left\|\phi_{R}^{\perp}\right\|_{L^{2}}^{2}, \quad d_{L^{2}\left(R^{+}\right)}^{2}\left(\phi_{I}, X_{1}\right)=\left\|\phi_{I}^{\perp}\right\|_{L^{2}}^{2} .
$$

By some straightforward computations, we have

$$
\begin{aligned}
& L_{1}\left(\phi_{R}, \phi_{R}\right)+L_{1}\left(\phi_{I}, \phi_{I}\right)+(\mu-1) \alpha_{R}\left(c_{R}^{2}+c_{I}^{2}\right) \int_{0}^{\infty} w_{y_{0}}^{2} d y \\
& \quad+(p-1)(\mu-1)^{2}\left(c_{R}^{2}+c_{I}^{2}\right) \int_{0}^{\infty} w_{y_{0}}^{p+1} d y \\
& \quad+\alpha_{R}\left(\left\|\phi_{R}^{\perp}\right\|_{L^{2}}^{2}+\left\|\phi_{I}^{\perp}\right\|_{L^{2}}^{2}\right)=0 .
\end{aligned}
$$

By Lemma 2.8 (2), we get 


$$
\begin{gathered}
(\mu-1) \alpha_{R}\left(c_{R}^{2}+c_{I}^{2}\right) \int_{0}^{\infty} w_{y_{0}}^{2} d y+(p-1)(\mu-1)^{2}\left(c_{R}^{2}+c_{I}^{2}\right) \\
\times \int_{0}^{\infty} w_{y_{0}}^{p+1} d y+\left(\alpha_{R}+a_{1}\right)\left(\left\|\phi_{R}^{\perp}\right\|_{L^{2}}^{2}+\left\|\phi_{I}^{\perp}\right\|_{L^{2}}^{2}\right) \leq 0 .
\end{gathered}
$$

Since $\mu>1$, we must have $\alpha_{R}<0$, which proves Theorem 2.9 in Case 1: $r=2,1<p \leq 3$.

Now we consider Case 2: $r=p+1,1<p<\infty$.

Then the nonlocal operator in (2.19) becomes

$$
L \phi=L_{0} \phi-\mu(p-1) \frac{\int_{0}^{\infty} w_{y_{0}}^{p} \phi d y}{\int_{0}^{\infty} w_{y_{0}}^{p+1} d y} w_{y_{0}}^{p} .
$$

We need to define yet another new operator:

$$
L_{2} \phi:=L_{0} \phi-(p-1) \frac{\int_{0}^{\infty} w_{y_{0}}^{p} \phi d y}{\int_{0}^{\infty} w_{y_{0}}^{p+1} d y} w_{y_{0}}^{p} .
$$

We have the following result.

Lemma 2.13. (1) $L_{2}$ is self-adjoint and the kernel of $L_{2}$ (denoted by $X_{2}$ ) is spanned by $w_{y_{0}}$.

(2) There exists a positive constant $c_{3}>0$ such that

$$
\begin{aligned}
L_{2}(\phi, \phi):= & \int_{0}^{\infty}\left[\left(\phi^{\prime}\right)^{2}+\phi^{2}-p w_{y_{0}}^{p-1} \phi^{2}\right] d y \\
& +\frac{(p-1)\left(\int_{0}^{\infty} w_{y_{0}}^{p} \phi d y\right)^{2}}{\int_{0}^{\infty} w_{y_{0}}^{p+1} d y} \\
\geq & c_{3} d_{L^{2}\left(R^{+}\right)}^{2}\left(\phi, X_{2}\right), \quad \forall \phi \in H_{\mathrm{rob}}^{1}\left(R^{+}\right) .
\end{aligned}
$$

Proof: The proof of (1) is similar to that of Lemma 2.8. We omit the details. It remains to prove (2). Suppose (2) is not true, then by (1) there exists $(\alpha, \phi)$ such that (i) $\alpha>0$, (ii) $\phi \perp w_{y_{0}}$, and (iii) $L_{2} \phi=\alpha \phi$.

We show that this is impossible. From (ii) and (iii), we have

$$
\left(L_{0}-\alpha\right) \phi=\frac{(p-1) \int_{0}^{\infty} w_{y_{0}}^{p} \phi d y}{\int_{0}^{\infty} w_{y_{0}}^{p+1} d y} w_{y_{0}}^{p}
$$

Similar to the proof of Lemma 2.8, we have that $\int_{0}^{\infty} w_{y_{0}}^{p} \phi d y$ $\neq 0$ and $\alpha \neq \mu_{1}$. Hence $L_{0}-\alpha$ is invertible. So (2.29) implies

$$
\phi=\frac{(p-1) \int_{0}^{\infty} w_{y_{0}}^{p} \phi d y}{\int_{0}^{\infty} w_{y_{0}}^{p+1} d y}\left(L_{0}-\alpha\right)^{-1} w_{y_{0}}^{p} .
$$

Thus

$$
\int_{0}^{\infty} w_{y_{0}}^{p} \phi d y=(p-1) \frac{\int_{0}^{\infty} w_{y_{0}}^{p} \phi d y}{\int_{0}^{\infty} w_{y_{0}}^{p+1} d y} \int_{0}^{\infty}\left[\left(L_{0}-\alpha\right)^{-1} w_{y_{0}}^{p}\right] w_{y_{0}}^{p} d y
$$

and

$$
\int_{0}^{\infty} w_{y_{0}}^{p+1} d y=(p-1) \int_{0}^{\infty}\left[\left(L_{0}-\alpha\right)^{-1} w_{y_{0}}^{p}\right] w_{y_{0}}^{p} d y .
$$

Let

$$
h_{2}(\alpha)=(p-1) \int_{0}^{\infty}\left[\left(L_{0}-\alpha\right)^{-1} w_{y_{0}}^{p}\right] w_{y_{0}}^{p} d y-\int_{0}^{\infty} w_{y_{0}}^{p+1} d y .
$$

Then we have

$$
h_{2}(0)=(p-1) \int_{0}^{\infty}\left(L_{0}^{-1} w_{y_{0}}^{p}\right) w_{y_{0}}^{p} d y-\int_{0}^{\infty} w_{y_{0}}^{p+1} d y=0 .
$$

Moreover, we compute

$$
\begin{aligned}
h_{2}^{\prime}(\alpha) & =(p-1) \int_{0}^{\infty}\left[\left(L_{0}-\alpha\right)^{-2} w_{y_{0}}^{p}\right] w_{y_{0}}^{p} d y \\
& =(p-1) \int_{0}^{\infty}\left[\left(L_{0}-\alpha\right)^{-1} w_{y_{0}}^{p}\right]^{2} d y>0 .
\end{aligned}
$$

This implies $h_{2}(\alpha)>0$ for all $\alpha \in\left(0, \mu_{1}\right)$. Clearly, also $h_{2}(\alpha)<0$ for $\alpha \in\left(\mu_{1}, \infty\right)$. This is a contradiction to (2.30) and the lemma is proved.

We now finish the proof of Theorem 2.9 in Case 2.

Let $\alpha_{0}=\alpha_{R}+i \alpha_{I}$ and $\phi=\phi_{R}+i \phi_{I}$. Since $\alpha_{0} \neq 0$, we can choose $\phi \perp \operatorname{kernel}\left(L_{0}\right)$. Then, similarly to Case 1 , we obtain the two equations

$$
\begin{gathered}
L_{0} \phi_{R}-(p-1) \mu \frac{\int_{0}^{\infty} w_{y_{0}}^{p} \phi_{R} d y}{\int_{0}^{\infty} w_{y_{0}}^{p+1} d y} w_{y_{0}}^{p}=\alpha_{R} \phi_{R}-\alpha_{I} \phi_{I}, \\
L_{0} \phi_{I}-(p-1) \mu \frac{\int_{0}^{\infty} w_{y_{0}}^{p} \phi_{I} d y}{\int_{0}^{\infty} w_{y_{0}}^{p+1} d y} w_{y_{0}}^{p}=\alpha_{R} \phi_{I}+\alpha_{I} \phi_{R} .
\end{gathered}
$$

Multiplying (2.31) by $\phi_{R}$ and (2.32) by $\phi_{I}$, integrating and adding, we obtain

$$
\begin{aligned}
-\alpha_{R} \int_{0}^{\infty}\left(\phi_{R}^{2}+\phi_{I}^{2}\right) d y & \\
= & L_{2}\left(\phi_{R}, \phi_{R}\right)+L_{2}\left(\phi_{I}, \phi_{I}\right) \\
& \quad+(p-1)(\mu-1) \frac{\left(\int_{0}^{\infty} w_{y_{0}}^{p} \phi_{R} d y\right)^{2}+\left(\int_{0}^{\infty} w_{y_{0}}^{p} \phi_{I} d y\right)^{2}}{\int_{0}^{\infty} w_{y_{0}}^{p+1} d y} .
\end{aligned}
$$

By Lemma 2.13 (2),

$$
\begin{aligned}
& \alpha_{R} \int_{0}^{\infty}\left(\phi_{R}^{2}+\phi_{I}^{2}\right)+a_{2} d_{L^{2}}^{2}\left(\phi, X_{1}\right) \\
& \quad+(p-1)(\mu-1) \frac{\left(\int_{0}^{\infty} w_{y_{0}}^{p} \phi_{R}\right)^{2}+\left(\int_{0}^{\infty} w_{y_{0}}^{p} \phi_{I}\right)^{2}}{\int_{0}^{\infty} w_{y_{0}}^{p+1}} \leq 0,
\end{aligned}
$$

which implies $\alpha_{R}<0$ since $\mu>1$.

Theorem 2.9 is thus proved in Case 2: $r=p+1,1<p$ $<\infty$.

Note that Theorem 2.9 implies Theorem 1.2, and Theorem 2.10 implies Theorem 1.3.

\section{EIGENVALUE ESTIMATES: PROOF OF THEOREM 1.1}

In this section, we shall study eigenvalue estimates for $L_{\epsilon}:=\epsilon^{2} \Delta-1+p\left(u_{\epsilon}\right)^{p-1}$, defined on $H_{\mathrm{rob}}^{1}(\Omega)$, in the case of an interior spike and finish the proof of Theorem 1.1. 
We will state a theorem for the small [i.e., $o(1)$ ] eigenvalues. But before we do this, let us first introduce some notation and give some important lemmas.

Let

$$
d \mu_{P_{0}}(z)=\lim _{\epsilon \rightarrow 0} \frac{e^{-2\left|z-P_{0}\right| / \epsilon} d z}{\int_{\partial \Omega} e^{-2\left|z-P_{0}\right| / \epsilon} d z} .
$$

It is easy to see that the support of $d \mu_{P_{0}}(z)$ is contained in $\bar{B}_{d\left(P_{0}, \partial \Omega\right)}\left(P_{0}\right) \cap \partial \Omega$.

A point $P_{0}$ is called a "nondegenerate peak point" if the following statements and hold: There exists $a \in R^{N}$ such that

$$
\int_{\partial \Omega} e^{\left\langle z-P_{0}, a\right\rangle}\left(z-P_{0}\right) d \mu_{P_{0}}(z)=0
$$

and the matrix

$$
\begin{aligned}
& {\left[\int_{\partial \Omega} e^{\left\langle z-P_{0}, a\right\rangle}\left(z-P_{0}\right)_{i}\left(z-P_{0}\right)_{j} d \mu_{P_{0}}(z)\right]} \\
& \quad:=G\left(P_{0}\right) \text { is nonsingular. }
\end{aligned}
$$

Such a vector $a$ is unique. Moreover, $G\left(P_{0}\right)$ is a positive definite matrix. A geometric characterization of a nondegenerate peak point $P_{0}$ is the following:

$$
P_{0} \in \text { interior }\left\{\text { convex hull of support }\left[d \mu_{P_{0}}(z)\right]\right\} .
$$

For a proof of the above, see Theorem 5.1 of Ref. 17.

Next, we introduce the following definition:

For each $P \in \Omega$, let $w_{\epsilon, P}$ be the unique solution of $\epsilon^{2} \Delta u-u+w^{p}\left(\frac{x-P}{\epsilon}\right)=0$ in $\Omega, \quad \epsilon \frac{\partial u}{\partial \nu}+a u=0$ on $\partial \Omega$.

Let $\varphi_{\epsilon, P}(x)=w\left(\frac{x-P}{\epsilon}\right)-w_{\epsilon, P}(x)$. Then $\varphi_{\epsilon, P}$ satisfies

$$
\begin{aligned}
& \epsilon^{2} \Delta \varphi_{\epsilon, P}-\varphi_{\epsilon, P}=0 \quad \text { in } \Omega, \\
& a \varphi_{\epsilon, P}+\epsilon \frac{\partial \varphi_{\epsilon, P}}{\partial \nu}=a w\left(\frac{x-P}{\epsilon}\right)+\epsilon \frac{\partial w\left(\frac{x-P}{\epsilon}\right)}{\partial \nu} \text { on } \partial \Omega .
\end{aligned}
$$

For $x \in \partial \Omega$, we have

$$
\begin{aligned}
& a w\left(\frac{x-P}{\epsilon}\right)+\epsilon \frac{\partial w\left(\frac{x-P}{\epsilon}\right)}{\partial \nu} \\
& \quad=a w\left(\frac{x-P}{\epsilon}\right)+w^{\prime}\left(\frac{x-P}{\epsilon}\right) \frac{\langle x-P, \nu\rangle}{|x-P|} \\
& \quad=w\left(\frac{x-P}{\epsilon}\right)\left[a-\frac{\langle x-P, \nu\rangle}{|x-P|}+O\left(\frac{\epsilon}{d(P, \partial \Omega)}\right)\right] \\
& \quad \geq(a-1-\delta) w\left(\frac{x-P}{\epsilon}\right),
\end{aligned}
$$

where $w^{\prime}(y)=\frac{d w(r)}{d r}$ for $r=|y|$ and $a-1-\delta>0$. Therefore, there exist two positive constants $C_{1}$ and $C_{2}$ such that

$$
C_{1} \varphi_{\epsilon, P, 1} \leq \varphi_{\epsilon, P} \leq C_{2} \varphi_{\epsilon, P, 1},
$$

where $\varphi_{\epsilon, P, 1}$ satisfies

$$
\begin{aligned}
& \epsilon^{2} \Delta \varphi_{\epsilon, P, 1}-\varphi_{\epsilon, P, 1}=0 \quad \text { in } \Omega, \\
& \varphi_{\epsilon, P, 1}+a^{-1} \epsilon \frac{\partial \varphi_{\epsilon, P, 1}}{\partial \nu}=w\left(\frac{x-P}{\epsilon}\right) \text { on } \partial \Omega .
\end{aligned}
$$

The study of $\varphi_{\epsilon, P, 1}$ depends on the following lemma.

Lemma 3.1 (theorem 3.8 of Ref. 18). Suppose that $d(P, \partial \Omega)>d_{0}>0$. Let $\varphi_{\epsilon, P}^{D}$ be the unique solution of

$$
\begin{gathered}
\epsilon^{2} \Delta \varphi_{\epsilon, P}^{D}-\varphi_{\epsilon, P}^{D}=0 \quad \text { in } \Omega, \\
\varphi_{\epsilon, P}^{D}=w\left(\frac{x-P}{\epsilon}\right) \quad \text { on } \partial \Omega .
\end{gathered}
$$

Then for any arbitrarily small $\delta>0$, the following holds for $\epsilon$ sufficiently small:

$$
\left|\epsilon \frac{\partial \varphi_{\epsilon, P}^{D}}{\partial \nu}\right| \leq(1+\delta) \varphi_{\epsilon, P}^{D} .
$$

From Lemma 3.1 we conclude that on $\partial \Omega$,

$$
\begin{aligned}
\varphi_{\epsilon, P}^{D}+a^{-1} \epsilon \frac{\varphi_{\epsilon, P}^{D}}{\partial \nu} & \leq \varphi_{\epsilon, P}^{D}\left[1+a^{-1}(1+\delta)\right] \\
& \leq\left[1+a^{-1}(1+\delta)\right] w\left(\frac{x-P}{\epsilon}\right)
\end{aligned}
$$

and

$$
\begin{aligned}
\varphi_{\epsilon, P}^{D}+a^{-1} \epsilon \frac{\varphi_{\epsilon, P}^{D}}{\partial \nu} & \geq \varphi_{\epsilon, P}^{D}\left[1-a^{-1}(1-\delta)\right] \\
& \geq\left[1-a^{-1}(1-\delta)\right] w\left(\frac{x-P}{\epsilon}\right) .
\end{aligned}
$$

Using a comparison principle, it is straightforward to derive the following lemma:

Lemma 3.2. There exist two positive constants $C_{1}$ and $C_{2}$ such that

$$
C_{1} \varphi_{\epsilon, P}^{D} \leq \varphi_{\epsilon, P, 1} \leq C_{2} \varphi_{\epsilon, P}^{D} .
$$

The convergence of (3.8) is well understood. It is studied in Sec. 4 of Ref. 19. By Lemma 4.6 of Ref. 19, we have the following convergence results:

Lemma 3.3. (i) Let $V_{\epsilon}(y):=\varphi_{\epsilon, x_{\epsilon}}\left(x_{\epsilon}+\epsilon y\right) / \varphi_{\epsilon, x_{\epsilon}}\left(x_{\epsilon}\right)$. Then $V_{\epsilon}(y) \rightarrow V_{0}(y)$ locally, where $V_{0}(y)$ is a solution of

$$
\Delta u-u=0, \quad u(0)=1, \quad u>0 \text { in } R^{N} .
$$

Moreover, for any $\sigma>0$,

$$
\sup _{y \in \Omega_{\epsilon}} e^{-(1+\sigma)|y|}\left[V_{\epsilon}(y)-V_{0}(y)\right] \rightarrow 0 .
$$

(ii) As $\epsilon \rightarrow 0$,

$-\epsilon \log \left[\varphi_{\epsilon, x_{\epsilon}}\left(x_{\epsilon}\right)\right] \rightarrow 2 d\left(x_{0}, \partial \Omega\right)$.

For $P \in \Omega$, let

$\Omega_{\epsilon, P}=\{y \mid \epsilon y+P \in \Omega\}$, 


$$
\begin{aligned}
& S_{\epsilon}(u)=\Delta u-u+u^{p} \quad \text { for } u \in H_{\mathrm{rob}}^{1}\left(\Omega_{\epsilon, P}\right), \quad \partial_{j}=\frac{\partial}{\partial P_{j}}, \\
& \mathcal{K}_{\epsilon, P}=\operatorname{span}\left\{\partial_{j} w_{\epsilon, P} \mid j=1, \ldots, N\right\} \subset H_{\mathrm{rob}}^{1}\left(\Omega_{\epsilon, P}\right), \\
& \mathcal{K}_{\epsilon, P}^{\perp}=\left\{u \in H_{\mathrm{rob}}^{1}\left(\Omega_{\epsilon, P}\right) \mid \int_{\Omega} u \partial_{j} w_{\epsilon, P}=0, j=1, \ldots, N\right\},
\end{aligned}
$$

and

$$
\begin{aligned}
& \mathcal{C}_{\epsilon, P}=\operatorname{span}\left\{\partial_{j} w_{\epsilon, P} \mid j=1, \ldots, N\right\} \subset L^{2}\left(\Omega_{\epsilon, P}\right), \\
& \mathcal{C}_{\epsilon, P}^{\perp}=\left\{u \in L^{2}\left(\Omega_{\epsilon, P}\right) \mid \int_{\Omega} u \partial_{j} w_{\epsilon, P}=0, j=1, \ldots, N\right\} .
\end{aligned}
$$

Let $Q_{\epsilon}^{0}:=P_{0}+\epsilon(1 / 2) d\left(P_{0}, \partial \Omega\right) a$, where $P_{0}$ is a nondegenerate peak-point [i.e., it satisfies Eqs. (3.2) and (3.3)] and $\Lambda:=B_{\beta_{0} \epsilon}\left(Q_{\epsilon}^{0}\right)$, where $\beta_{0}$ is sufficiently small.

For each $P \in \Lambda$ we can find a solution $\varphi_{\epsilon, P} \in \mathcal{K}_{\epsilon, P}^{\perp}$ such that

$$
S_{\epsilon}\left(w_{\epsilon, P}+\varphi_{\epsilon, P}\right) \in \mathcal{C}_{\epsilon, P}
$$

as was shown in Ref. 20.

Now we state our theorem on the small eigenvalues.

Theorem 3.4. The eigenvalue problem

$$
\begin{aligned}
& \epsilon^{2} \Delta \phi-\phi+p u_{\epsilon}^{p-1} \phi=\tau^{\epsilon} \phi \quad \text { in } \Omega, \\
& \epsilon \frac{\partial \phi}{\partial \nu}+a \phi=0 \quad \text { on } \partial \Omega
\end{aligned}
$$

admits the following set of o(1) eigenvalues:

$$
\tau_{j}^{\epsilon}=\left[c_{0}+o(1)\right] \varphi_{\epsilon, P_{0}}\left(P_{0}\right) \lambda_{j}, \quad j=1, \ldots, N,
$$

where $\lambda_{j}, j=1, \ldots, N$, are the eigenvalues of the matrix $G\left(P_{0}\right)$ introduced in Eq. (3.3) and

$$
c_{0}=2 d^{-2}\left(P_{0}, \partial \Omega\right) \frac{\int_{R^{N} p w^{p-1} w^{\prime} V_{0}^{\prime}(r) d y}}{\int_{R^{N}}\left(\frac{\partial w}{\partial y_{1}}\right)^{2} d y}<0,
$$

where $V_{0}(r)$ is the unique radial solution of the problem (3.10). Furthermore, the eigenfunction (suitably normalized) corresponding to $\tau_{j}^{\epsilon}, j=1, \ldots, N$, is given by

$$
\phi_{j}^{\epsilon}=\left.\sum_{l=1}^{N}\left[a_{j, l}+o(1)\right] \epsilon \frac{\partial w_{\epsilon, P}}{\partial P_{l}}\right|_{P=P_{\epsilon}},
$$

where $\vec{a}_{j}=\left(a_{j, 1}, \ldots, a_{j, N}\right)^{t}$ is the eigenvector of $G\left(P_{0}\right)$ corresponding to $\lambda_{j}$, namely

$$
G\left(P_{0}\right) \vec{a}_{j}=\lambda_{j} \vec{a}_{j}, \quad j=1, \ldots, N
$$

Proof of Theorem 3.4: Let

$$
u_{\epsilon}=w_{\epsilon, Q_{\epsilon}}+v_{\epsilon, Q_{\epsilon}}
$$

Let $\left(\tau^{\epsilon}, \phi_{\epsilon}\right)$ be a pair such that

$$
L_{\epsilon} \phi_{\epsilon}=\tau^{\epsilon} \phi_{\epsilon} \quad \text { in } \Omega, \quad \epsilon \frac{\partial \phi_{\epsilon}}{\partial \nu}+a \phi=0 \quad \text { on } \partial \Omega \text {. }
$$

We normalize $\phi_{\epsilon}$ such that $\left\|\phi_{\epsilon}\right\|_{\epsilon}=1$.

We now assume that $\tau_{\epsilon} \rightarrow 0$ as $\epsilon \rightarrow 0$. Then, after a scaling and limiting process (see Refs. 19, 21, and 22), we have $\widetilde{\phi}_{\epsilon}(y)=\phi_{\epsilon}\left(Q_{\epsilon}+\epsilon y\right) \rightarrow \phi_{0}$, where $\phi_{0}$ is a solution of

$$
\Delta v-v+p w^{p-1} v=0 \quad \text { in } R^{N}, \quad v \in H^{1}\left(R^{N}\right) .
$$

By Lemma 4.2 of Ref. 22, there exists $s_{j}$ such that $\phi_{0}$ $=\sum_{j=1}^{N} s_{j} \frac{\partial w}{\partial y_{j}}$.

This suggests that we decompose $\phi_{\epsilon}$ as $\phi_{\epsilon}$ $=\sum_{j=1}^{N} s_{j}^{\epsilon} \epsilon \partial_{j} w_{\epsilon, Q_{\epsilon}}+\bar{\phi}_{\epsilon}$, where $\bar{\phi}_{\epsilon} \in \mathcal{K}_{\epsilon, Q_{\epsilon}}^{\perp}$ and $\left|s_{j}^{\epsilon}\right| \leq C$. Since $\left\|\phi_{\epsilon}\right\|_{\epsilon}=1$, we have $\left\|\bar{\phi}_{\epsilon}\right\|_{\epsilon} \leq C$ and $\bar{\phi}_{\epsilon}$ satisfies

$$
\begin{aligned}
& \left(L_{\epsilon}-\tau^{\epsilon}\right) \bar{\phi}_{\epsilon}+\sum_{j=1}^{N} s_{j}^{\epsilon}\left[p\left(u_{\epsilon}\right)^{p-1} \epsilon \partial_{j} w_{\epsilon, Q_{\epsilon}}-p w^{p-1} \epsilon \partial_{j} w\right] \\
& =\tau^{\epsilon} \sum_{j=1}^{N} s_{j}^{\epsilon} \epsilon \partial_{j} w_{\epsilon, Q_{\epsilon}} .
\end{aligned}
$$

Since $\tau^{\epsilon} \rightarrow 0$, then by the same argument as in Proposition 6.3 of Ref. 23 we have that $\pi_{\epsilon, Q_{\epsilon}}{ }^{\circ}\left(L_{\epsilon}-\tau^{\epsilon}\right): \mathcal{K}_{\epsilon, Q_{\epsilon}}^{\perp} \rightarrow \mathcal{C}_{\epsilon, Q_{\epsilon}}^{\perp}$ is invertible. Since $\bar{\phi}_{\epsilon} \in \mathcal{K}_{\epsilon, Q_{\epsilon}}^{\perp}$, we have

$$
\begin{aligned}
& \left\|\bar{\phi}_{\epsilon}\right\|_{H^{1}\left(\Omega_{\epsilon, Q_{\epsilon}}\right)} \\
& =O\left(\left\|\sum_{j=1}^{N} s_{j}^{\epsilon}\left[p\left(u_{\epsilon}\right)^{p-1} \epsilon \partial_{j} w_{\epsilon, Q_{\epsilon}}-p w^{p-1} \epsilon \partial_{j} w\right]\right\|_{L^{2}\left(\Omega_{\left.\epsilon, Q_{\epsilon}\right)}\right.}\right) \\
& =O\left(\left[\left|\varphi_{\epsilon, Q_{\epsilon}}\left(Q_{\epsilon}\right)\right|^{(1+\sigma) / 2}\right] \sum_{j=1}^{N}\left|s_{j}^{\epsilon}\right|\right) .
\end{aligned}
$$

Multiplying (3.17) by $\epsilon \partial_{k}\left(w_{\epsilon, Q_{\epsilon}}\right)$ and integrating, we obtain

$$
\begin{aligned}
& \sum_{j=1}^{N} s_{j}^{\epsilon} \int_{\Omega_{\epsilon, Q_{\epsilon}}}\left[p\left(u_{\epsilon}\right)^{p-1} \epsilon \partial_{j} w_{\epsilon, Q_{\epsilon}}-p w^{p-1} \epsilon \partial_{j} w\right] \epsilon \partial_{k} w_{\epsilon, Q_{\epsilon}} d x \\
& =\tau^{\epsilon} \sum_{j=1}^{N} \int_{\Omega_{\epsilon, Q_{\epsilon}}} s_{j}^{\epsilon} \epsilon \partial_{j} w_{\epsilon, Q_{\epsilon}} \epsilon \partial_{k} w_{\epsilon, Q_{\epsilon}} d x \\
& \quad+\int_{\Omega_{\epsilon, Q_{\epsilon}}}\left[p\left(u_{\epsilon}\right)^{p-1} \bar{\phi}_{\epsilon} \epsilon \partial_{k}\left(w_{\epsilon, Q_{\epsilon}}\right)-p w^{p-1} \bar{\phi}_{\epsilon} \epsilon \partial_{k} w\right] d x \\
& \quad+O\left(\mid \tau_{\epsilon}\left\|\bar{\phi}_{\epsilon}\right\|_{H^{1}\left(\Omega_{\epsilon, Q_{\epsilon}}\right)}\right) .
\end{aligned}
$$

We first estimate the left-hand side of (3.18). To begin with, we calculate 


$$
\begin{aligned}
& -\int_{\Omega_{\epsilon, Q_{\epsilon}}}\left[\left.p w^{p-1} \epsilon \frac{\partial w}{\partial P_{j}}\right|_{P=Q_{\epsilon}}-\left.p\left(w_{\epsilon, Q_{\epsilon}}+v_{\epsilon, Q_{\epsilon}}\right)^{p-1} \epsilon \frac{\partial w_{\epsilon, P}}{\partial P_{k}}\right|_{P=Q_{\epsilon}}\right] d y \\
& =-\left.\epsilon^{2} \int_{\Omega_{\epsilon, Q_{\epsilon}}}\left[\left.p w^{p-1} \frac{\partial w}{\partial P_{j}}\right|_{P=Q_{\epsilon}}-\left.p\left(w_{\epsilon, Q_{\epsilon}}\right)^{p-1} \frac{\partial w_{\epsilon, P}}{\partial P_{j}}\right|_{P=Q_{\epsilon}}\right] \frac{\partial w_{\epsilon, P}}{\partial P_{k}}\right|_{P=Q_{\epsilon}} d y+O\left[\left|\varphi_{\epsilon, Q_{\epsilon}}\left(Q_{\epsilon}\right)\right|^{1+\sigma}\right] \\
& =-\left.\left.\epsilon^{2} \int_{\Omega_{\epsilon, Q_{\epsilon}}} \frac{\partial}{\partial P_{j}}\right|_{P=Q_{\epsilon}}\left[w^{p}-\left(w_{\epsilon, Q_{\epsilon}}\right)^{p}\right] \frac{\partial w_{\epsilon, P}}{\partial P_{k}}\right|_{P=Q_{\epsilon}} d y+O\left[\left|\varphi_{\epsilon, Q_{\epsilon}}\left(Q_{\epsilon}\right)\right|^{1+\sigma}\right] \\
& =-\left.\left.\epsilon^{2} \int_{\Omega_{\epsilon, Q_{\epsilon}}} \frac{\partial}{\partial P_{j}}\right|_{P=Q_{\epsilon}}\left[p w^{p-1} \varphi_{\epsilon, Q_{\epsilon}}\left(Q_{\epsilon}+\epsilon y\right)\right] \frac{\partial w_{\epsilon, P}}{\partial P_{k}}\right|_{P=Q_{\epsilon}}+O\left[\left|\varphi_{\epsilon, Q_{\epsilon}}\left(Q_{\epsilon}\right)\right|^{1+\sigma}\right] \\
& =2 \varphi_{\epsilon, P_{0}}\left(P_{0}\right)[1+o(1)] \int_{R^{N}} p w^{p-1} \int_{\partial \Omega} e^{\left\langle z-P_{0}|| z-P_{0} \mid, y\right\rangle} e^{\left\langle z-P_{0}\left|z-P_{0}\right|, 2\left(Q_{\epsilon}-P_{0}\right) / \epsilon\right\rangle} d \mu_{P_{0}}(z)\left(\frac{z-P_{0}}{\left|z-P_{0}\right|}\right) \frac{\partial w}{\partial y_{k}} d y+O\left[\left|\varphi_{\epsilon, Q_{\epsilon}}\left(Q_{\epsilon}\right)\right|^{1+\sigma}\right] \\
& =2 \varphi_{\epsilon, P_{0}}\left(P_{0}\right)[1+o(1)] \int_{R^{N}} p w^{p-1} \int_{\partial \Omega} e^{\left\langle z-P_{0}|| z-P_{0} \mid, y\right\rangle} e^{\left\langle z-P_{0}, a\right\rangle}\left(\frac{z-P_{0}}{\left|z-P_{0}\right|}\right) d \mu_{j}(z) \frac{\partial w}{\partial y_{k}} d y+O\left[\left|\varphi_{\epsilon, Q_{\epsilon}}\left(Q_{\epsilon}\right)\right|^{1+\sigma}\right] \\
& =\frac{2 \gamma}{d^{2}\left(P_{0}, \partial \Omega\right)} \varphi_{\epsilon, P_{0}}\left(P_{0}\right)\left(\int_{\partial \Omega} e^{\left\langle z-P_{0}, a\right\rangle}\left(z-P_{0}\right)_{i}\left(z-P_{0}\right)_{k} d \mu_{P_{0}}(z)+o(1)\right),
\end{aligned}
$$

where

$$
\gamma:=\int_{R^{N}} p w^{p-1} w^{\prime}(y) V_{0}^{\prime}(y) d y .
$$

For the left-hand side of (3.18), we have

$$
\begin{aligned}
\text { 1.h.s. of (3.16) } & =\sum_{j=1}^{N} s_{j}^{\epsilon}\left(\int_{\Omega_{\epsilon, Q_{\epsilon}}}\left[p\left(w_{\epsilon, Q_{\epsilon}}\right)^{p-1} \epsilon \partial_{j} w_{\epsilon, Q_{\epsilon}}-p w^{p-1} \epsilon \partial_{j} w\right] \epsilon \partial_{k} w_{\epsilon, Q_{\epsilon}} d y\right)+O\left[\left|\varphi_{\epsilon, Q_{\epsilon}}\left(Q_{\epsilon}\right)\right|^{1+\sigma}\right] \\
& =\int_{\partial \Omega} e^{\left\langle z-P_{0}, a\right\rangle}\left\langle\frac{z-P_{0}}{\left|z-P_{0}\right|}, s^{\epsilon}\right\rangle\left(\frac{z-P_{0}}{\left|z-P_{0}\right|}\right)_{k} \varphi_{\epsilon, P_{0}}\left(P_{0}\right) d \mu_{P_{0}}(z)[-2 \gamma+o(1)]
\end{aligned}
$$

where $s^{\epsilon}=\left(s_{1}^{\epsilon}, \ldots, s_{N}^{\epsilon}\right)$.

Similar but simpler computations for the right-hand side of (3.18) give

$$
\begin{aligned}
\text { r.h.s. of (3.16) }= & \tau^{\epsilon} \sum_{j=1}^{N} s_{j}^{\epsilon}\left[B \delta_{j k}+o(1)\right] \\
& +O\left(\sum_{j=1}^{N}\left|s_{j}^{\epsilon}\right|\left|\varphi_{\epsilon, Q_{\epsilon}}\left(Q_{\epsilon}\right)\right|^{(1+\sigma)}\right) \\
& +O\left[\left|\tau^{\epsilon}\right|\left(\sum_{j=1}^{N}\left|s_{j}^{\epsilon}\right|\left|\varphi_{\epsilon, Q_{\epsilon}}\left(Q_{\epsilon}\right)\right|^{(1+\sigma) / 2}\right)\right],
\end{aligned}
$$

where $B=\int_{R^{N}}\left(\frac{\partial w}{\partial y_{1}}\right)^{2} d y$.

Hence we have

$$
\left|\tau^{\epsilon}\right|=O\left[\varphi_{\epsilon, Q_{\epsilon}}\left(Q_{\epsilon}\right)\right]=O\left[\varphi_{\epsilon, P_{0}}\left(P_{0}\right)\right]
$$

and $\tau^{\epsilon} / \varphi_{\epsilon, P_{0}}\left(P_{0}\right) \rightarrow \tau_{0}, s^{\epsilon} \rightarrow s$, where $\left(\tau_{0}, s\right)$ satisfies

$$
(-2 \gamma) G\left(P_{0}\right) s=B d^{2}\left(P_{0}, \partial \Omega\right) \tau_{0} s .
$$

Thus $\frac{B d^{2}\left(P_{0}, \partial \Omega\right)}{-2 \gamma} \tau_{0}$ is an eigenvalue of $G\left(P_{0}\right)$. Therefore, $\tau^{\epsilon} / \varphi_{\epsilon, P_{0}}\left(P_{0}\right) \stackrel{-2 \gamma}{\rightarrow} \tau_{j}, s^{\epsilon} \rightarrow \vec{a}_{j}$, where

$$
\tau_{j}=\frac{-2 \gamma}{B d^{2}\left(P_{0}, \partial \Omega\right)} \lambda_{j}, \quad G\left(P_{0}\right) \vec{a}_{j}=\lambda_{j} \vec{a}_{j}, \quad j=1, \ldots, N .
$$

By an argument of Dancer, ${ }^{15}$ we know that these are the only small eigenvalues of the order $o(1)$ as $\epsilon \rightarrow 0$.

This finishes the proof of Theorem 3.4.

\section{Completion of the Proof of Theorem 1.1:}

The small eigenvalues given by Theorem 3.4 all have a negative real part. By a proof along the lines of the proof of Theorem 2.9 (replacing $w_{y_{0}}$ by $w$ and considering interior spikes instead of near-boundary spikes), the large eigenvalues all have negative real part. Finally, Theorem 1.1 follows by combining these two results.

\section{NUMERICAL SIMULATIONS}

We show numerical simulations that display the various effects that have been analytically proved in this paper.

We consider the Gierer-Meinhardt system (1.1) and (1.2) on $\Omega=(-1,1)$ for the following parameters: diffusion constants $\epsilon^{2}=0.01, D=10^{9}$, time relaxation constant $\tau=10^{-9}$, Robin boundary condition parameters varying $a_{A}, a_{H}=0$, reaction constants varying $p$ and $q, r=2, s=0$. 
First we consider the classical Gierer-Meinhardt system with $p=2, q=1$. We show stable near-boundary spikes for various $a_{A}$ (Fig. 1) and interior spikes for various $a_{A}$ (Fig. 2). We see that a change of $a_{A}$ has a strong influence on a nearboundary spike, but only a minor influence on an interior spike.

Then we numerically explore the instability in the dynamics of near-boundary spikes. We consider the GiererMeinhardt system for various $p, q=2, r=2, s=0$ with the Robin boundary condition for $a_{A}=0.8$. We start with $p=4.0$ and then increase $p$ incrementally in steps of 0.01 . The final steady state (stable stationary near-boundary spike) for the previous $p$ is used as an initial condition for the next one. The final steady state is displayed for $p=4.5,4.8$, and 4.85 (Fig. 3).

At $p=4.86$, a rather dramatic change of stability is observed: The solution oscillates with large frequencies (Fig. 4). The simulations show a sharp peak, and the simulation breaks down: The amplitudes of the solution become very large, and the finite-element software is no longer able to resolve the solution since this peak has a very high amplitude appearing on a very small spatial scale. This is similar to phenomena that occur for supercritical systems. In some sense the Robin boundary condition is able to squeeze the threshold between sub- and supercritical behavior to lower reaction rates that corresponds to the formula $\mu_{0}(a)>1$.

Comparing the threshold $p=4.86$ with the theoretical results, one could first test if the instability comes from the threshold $a=a_{0}(p)$ given in Proposition 2.5. The asymptotic result in Remark 2.6 implies that for $p=4.86$ we get $a_{0}(p)$ $\sim 0.11$, which is far from the numerical value $a=0.8$. On the other hand, we have $\mu=4 / 3.86-1.04$, which is larger than 1 but very close to 1 . So the instability here comes from the threshold given by $\mu<1.04$. We also make the interesting numerical observation that even if $a$ is far away from $a_{0}(p)$, the threshold $\mu_{0}(a)$ can be very close to 1 . This implies that one has to be very careful in numerical simulations in order not to miss this extended but small parameter range of instability that is stated in Theorem 1.3.

\section{DISCUSSION}

This paper is just the start of our research program into the role of boundary conditions for reaction-diffusion systems. We have only considered the shadow system of the Gierer-Meinhardt system for the Robin boundary condition. It is also important to investigate the influence of the Robin boundary condition for general reaction-diffusion systems, starting with the full Gierer-Meinhardt system.

More specifically, for the full Gierer-Meinhardt system (1.1) in one space dimension an important issue is understanding the role of the Robin boundary condition for the inhibitor $H$, whereas in this paper we have only investigated the Robin boundary condition for the activator $A$. How does the Hopf bifurcation threshold change if Robin boundary conditions are put on $H$ ? What can be said about positive eigenvalues? This is also an interesting question for the shadow system.
As $a$ approaches 1, by Theorem A the minimal energy solution changes from being a near-boundary spike to being an interior spike. How can this change of behavior be analyzed in the case in which $a$ is exponentially close to $a$ and this changeover takes place, in particular with respect to a stability analysis?

Multiple spikes are another interesting topic. Contrary to Dirichlet or Neumann boundary conditions, for Robin boundary conditions one does not have spikes of equal amplitudes and distances and even the existence is a challenging question.

Higher-dimensional problems will be addressed in the future, and they are naturally very important for biological applications. We hope to gain a better understanding of the processes that are behind the selection of some relevant patterns out of the many possible patterns in a reaction-diffusion system. Here the boundary conditions play a major role and stability analysis seems to be the appropriate mathematical tool to address these issues in a rigorous framework.

\section{ACKNOWLEDGMENTS}

The work of J.W. is supported by a Grant from RGC of Hong Kong. The work of M.W. is supported by a BRIEF Award from Brunel University. P.K.M. and M.W. thank the Department of Mathematics at CUHK for their kind hospitality. We thank two anonymous referees who helped us to improve the paper.

${ }^{1}$ R. Dillon, P. K. Maini, and H. G. Othmer, J. Math. Biol. 32, 345 (1994).

${ }^{2}$ A. M. Turing, Philos. Trans. R. Soc. London, Ser. B 237, 37 (1952).

${ }^{3}$ A. Gierer and H. Meinhardt, J. Funct. Anal. 12, 30 (1972).

${ }^{4}$ H. Meinhardt, Models of Biological Pattern Formation (Academic Press, London, 1982).

${ }^{5} \mathrm{H}$. Meinhardt, The Algorithmic Beauty of Sea Shells, 2nd ed. (Springer, Berlin, 1998).

${ }^{6}$ D. Iron, M. Ward, and J. Wei, Physica D 150, 25 (2001).

${ }^{7}$ M. J. Ward and J. Wei, Eur. J. Appl. Math. 13, 283 (2002).

${ }^{8}$ M. J. Ward and J. Wei, J. Nonlinear Sci. 13, 209 (2003).

${ }^{9}$ M. J. Ward and J. Wei, Eur. J. Appl. Math. 14, 677 (2003).

${ }^{10}$ J. Wei and M. Winter, SIAM J. Math. Anal. 30, 1241 (1999).

${ }^{11}$ J. Wei and M. Winter, J. Differ. Equations 178, 478 (2002).

${ }^{12}$ J. Wei and M. Winter, J. Nonlinear Sci. 11, 415 (2001).

${ }^{13}$ J. Wei, Eur. J. Appl. Math. 10, 353 (1999).

${ }^{14}$ J. Wei, Proc. - R. Soc. Edinburgh, Sect. A: Math. 131, 1457 (2001).

${ }^{15}$ E. N. Dancer, Methods Appl. Anal. 8, 245 (2001).

${ }^{16}$ C.-S. Lin and W.-M. Ni, in Calculus of Variations and Partial Differential Equations, Lecture Notes in Math. Vol. 1340 (Springer, Berlin, 1988), p. 160.

${ }^{17}$ J. Wei, Tohoku Math. J. 50, 159 (1998).

${ }^{18}$ J. Wei, Diff. Integral Eq. 13, 15 (2000).

${ }^{19}$ W.-M. Ni and J. Wei, Commun. Pure Appl. Math. 48, 731 (1995).

${ }^{20}$ H. Berestycki and J. Wei, Ann. Scuola Norm. Sup. Pisa, Cl. Sci. 5, 199 (2003).

${ }^{21}$ W.-M. Ni and I. Takagi, Commun. Pure Appl. Math. 41, 819 (1991).

${ }^{22}$ W.-M. Ni and I. Takagi, Duke Math. J. 70, 247 (1993).

${ }^{23}$ J. Wei, Proc. - R. Soc. Edinburgh, Sect. A: Math. 128, 849 (1998).

${ }^{24}$ A. Doelman, R. A. Gardner, and T. J. Kaper, Physica D 122, 1 (1998).

${ }^{25}$ A. Doelman and R. A. Gardner, Indiana Univ. Math. J. 50, 443 (2001).

${ }^{26}$ A. Doelman, T. J. Kaper, and H. van der Ploeg, Methods Appl. Anal. 8, $387(2001)$.

${ }^{27}$ A. Doelman, T. J. Kaper, and P. A. Zegeling, Nonlinearity 10, 523 (1997).

${ }^{28}$ J. Wei, Int. J. Bifurcation Chaos Appl. Sci. Eng. 10, 1485 (2000).

${ }^{29}$ J. Wei and M. Winter, Int. J. Bifurcation Chaos Appl. Sci. Eng. 13, 1529 (2003).

${ }^{30}$ J. Wei and L. Zhang, Ann. Scuola Norm. Sup. Pisa, Cl. Sci. 30, 41 (2001). 